\title{
Low mutational load and high mutation rate variation in gut commensal bacteria
}

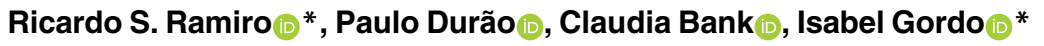 \\ Instituto Gulbenkian de Ciência, Oeiras, Portugal \\ * rramiro@ igc.gulbenkian.pt (RSR); igordo @igc.gulbenkian.pt (IG)
}

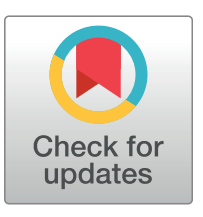

\section{Gopenaccess}

Citation: Ramiro RS, Durão P, Bank C, Gordo I (2020) Low mutational load and high mutation rate variation in gut commensal bacteria. PLOS Biol 18 (3): e3000617. https://doi.org/10.1371/journal. pbio. 3000617

Academic Editor: Nick H. Barton, Institute of Science and Technology Austria (IST Austria), AUSTRIA

Received: March 5, 2019

Accepted: February 5, 2020

Published: March 10, 2020

Copyright: ๑ 2020 Ramiro et al. This is an open access article distributed under the terms of the Creative Commons Attribution License, which permits unrestricted use, distribution, and reproduction in any medium, provided the original author and source are credited.

Data Availability Statement: Data and code can be downloaded from the figshare repository: https:// doi.org/10.6084/m9.figshare.11409528 and https://doi.org/10.6084/m9.figshare.10048427. Whole-genome sequencing data can be downloaded from NCBI (http://www.ncbi.nlm.nih. gov/sra; accession no. PRJNA580181).

Funding: RSR and PD were supported by postdoctoral fellowships from Fundação para a Ciência e Tecnologia (www.fct.pt), Portugal (SFRH/ BPD/119110/2016 and SFRH/BPD/118474/2016,

\section{Abstract}

Bacteria generally live in species-rich communities, such as the gut microbiota. Yet little is known about bacterial evolution in natural ecosystems. Here, we followed the longterm evolution of commensal Escherichia coli in the mouse gut. We observe the emergence of mutation rate polymorphism, ranging from wild-type levels to 1,000-fold higher. By combining experiments, whole-genome sequencing, and in silico simulations, we identify the molecular causes and explore the evolutionary conditions allowing these hypermutators to emerge and coexist within the microbiota. The hypermutator phenotype is caused by mutations in DNA polymerase III proofreading and catalytic subunits, which increase mutation rate by approximately 1,000 -fold and stabilise hypermutator fitness, respectively. Strong mutation rate variation persists for $>1,000$ generations, with coexistence between lineages carrying 4 to $>600$ mutations. The in vivo molecular evolution pattern is consistent with fitness effects of deleterious mutations $s_{d} \leq 10^{-4} /$ generation, assuming a constant effect or exponentially distributed effects with a constant mean. Such effects are lower than typical in vitro estimates, leading to a low mutational load, an inference that is observed in in vivo and in vitro competitions. Despite large numbers of deleterious mutations, we identify multiple beneficial mutations that do not reach fixation over long periods of time. This indicates that the dynamics of beneficial mutations are not shaped by constant positive Darwinian selection but could be explained by other evolutionary mechanisms that maintain genetic diversity. Thus, microbial evolution in the gut is likely characterised by partial sweeps of beneficial mutations combined with hitchhiking of slightly deleterious mutations, which take a long time to be purged because they impose a low mutational load. The combination of these two processes could allow for the longterm maintenance of intraspecies genetic diversity, including mutation rate polymorphism. These results are consistent with the pattern of genetic polymorphism that is emerging from metagenomics studies of the human gut microbiota, suggesting that we have identified key evolutionary processes shaping the genetic composition of this community. 
respectively). IG is grateful for support from the University of Cologne Cooperation Agreement: Predictability in Evolution (CRC 1310) by the German Research Foundation (http://www.dfg.de/ en/) and Instituto Gulbenkian de Ciência/Fundação Calouste Gulbenkian (http://igc.gulbenkian.pt, http://gulbenkian.pt) and from the Global Grant for Gut Health, co-supported by Yakult and Nature Research. CB and IG are grateful for the support from JPIAMR/0001/2016 (PREPARE); Joint Programming Initiative on Antimicrobial Resistance (https://www.jpiamr.eu/); and Fundação para a Ciência e Tecnologia (www.fct.pt), Portugal. $\mathrm{CB}$ is grateful for support from the European Molecular Biology Organization for the Installation Grant IG4152 (http://embo.org/) and from a European Research Council Starting Grant 804569 - FIT2GO (https://erc.europa.eu/). This work was further supported by Congento LISBOA-01-0145FEDER-022170, cofinanced by FCT (Portugal) and Lisboa2020, under the PORTUGAL2020 agreement (European Regional Development Fund). This work was partially supported by ONEIDA project (LISBOA-01-0145-FEDER-016417) cofunded by FEEl (Fundos Europeus Estruturais e de Investimento) from "Programa Operacional Regional Lisboa 2020" and by national funds from FCT (Fundação para a Ciência e a Tecnologia). The funders had no role in study design, data collection and analysis, decision to publish, or preparation of the manuscript.

Competing interests: The authors have declared that no competing interests exist.

Abbreviations: CFP, cyan fluorescent protein; CFU, colony-forming units; $\mathrm{COG}$, cluster of orthologous genes; IS, insertion sequence; LB, lysogeny broth; MA, mutation accumulation; NFDS, negative frequency-dependent selection; ns, not significant; Rif $^{\mathrm{R}}$, rifampicin-resistant; SNP, single nucleotide polymorphism; YFP, yellow fluorescent protein.

\section{Introduction}

Bacteria typically live in multispecies ecosystems [1]. One of such communities is the human gut microbiota, which is key for host health and can host up to $10^{13}$ bacteria and hundreds of species. Over the past decade, extensive efforts have been made to characterise the factors (e.g., diet, antibiotics) that shape the species-level composition of this ecosystem (e.g., [2-4]). More recently, it has become clear that there can be abundant genetic diversity within each species colonising a given individual [5-8]. Importantly, strain level variation is key for multiple phenotypes ranging from antibiotic resistance and virulence to colonisation resistance (e.g., [911]). However, little is known about how different evolutionary mechanisms-such as mutation, recombination, migration, genetic drift, and natural selection-shape bacterial genetic diversity within the mammalian gut [12].

Mutation is the raw material for natural selection. The genomic mutation rate $(U)$ of DNAbased microbes is typically around 0.001 per generation $[13,14]$, suggesting that it is shaped by general evolutionary forces, independent of phylogeny or ecological niche $[13,14]$. Nevertheless, within-species variation of mutation rates can be observed in natural isolates of commensal and pathogenic bacteria (typically to increase mutation rate, i.e., mutator clones; e.g., $[8,15-18])$. Based on the known DNA repair mechanisms, mutators with 10 to $>10,000$-fold increases in mutation rate could, in principle, emerge and spread $[19,20]$. However, from the balance between the capacity to adapt (adaptability) and robustness at maintaining current fitness (adaptedness), theory predicts that mutator clones with an increase in mutation rate of 10- to 200-fold should be the most common (e.g., [21,22]; we refer to the fold-change in mutation rate of a mutator clone as its mutator strength). Indeed, when mutator emergence was observed in vitro, very strong mutators ( $>300$-fold) were rarely detected [23-25]. However, in in vitro experiments, a bacterial lineage evolves in the absence of the multitude of biotic interactions that occur in natural ecosystems, such as the mammalian gut microbiota. In this ecosystem, biotic interactions can rapidly fluctuate $[26,27]$ because of changes in community composition or migration of novel lineages, often caused by community perturbations such as dietary changes or antibiotic intake $[2,3,28]$. Under fluctuating environments, theory predicts that mutator alleles can increase in frequency, either reaching fixation or leading to mutation rate polymorphism [22,29]. Yet knowledge of within-host mutation rate variation [15-18,30] and its temporal dynamics within a diverse multispecies community [8] remains limited.

Here, we sought to gain a better understanding of the evolutionary forces shaping bacterial evolution in the mammalian gut. We followed the long-term evolution of a new commensal strain of E. coli as it invaded and established itself in the complex ecosystem of the mouse gut after a perturbation caused by antibiotic treatment. Using an experimental evolution approach, we tracked the evolution of two defined E. coli lineages in real time. During this experiment, we observed the coexistence of lineages with mutation rates ranging from wild-type levels to 1,000 -fold higher. We determine the molecular causes and suggest the evolutionary conditions that allow such strong mutators to emerge and persist within a complex microbiota. At the molecular level, we show that mutations in the proofreading $(\alpha)$ and catalytic $(\varepsilon)$ subunits of DNA polymerase III cause a 1,000-fold mutation rate increase and improve hypermutator fitness, respectively. At the evolutionary level, theoretical simulations suggest that the population dynamics of the mutator lineages and their pattern of molecular evolution are compatible with deleterious mutations of weak effect. Such estimates for the in vivo mutational effects are lower than current estimates from in vitro mutation accumulation (MA) experiments (0.001 to 0.03 per generation [31-35]). This indicates that the mutational load may be lower in the mouse gut than in vitro-a result that we experimentally validate. Despite the large number of deleterious mutations observed in the mutators, we can identify multiple beneficial mutations. 
However, no fixations were detected for specific alleles or at the functional level (gene/operon). This suggests that the trajectories of most beneficial mutations are shaped by mechanisms such as negative-frequency dependence, temporally fluctuating selection, or metabolic/spatial niche partitioning (rather than constant positive Darwinian selection). The data reveal that the evolution of commensal bacteria within the mammalian gut is consistent with both the nearly neutral theory of molecular evolution and with the theory of genetic draft $[36,37]$. The first poses that most spontaneous mutations are very slightly deleterious, and the second proposes that the fate of these mutations is determined by their ability to hitchhike with linked beneficial mutations, which continuously increase in frequency.

\section{Results and discussion}

\section{Emergence and maintenance of mutation rate variation in a gut commensal strain}

To be able to study the evolution of new E. coli strains as they invade and colonise the mouse gut, we used a short-course antibiotic treatment (8 days with streptomycin), which perturbs microbiota composition and leads to a decrease in its species diversity (including killing native E. coli). With this setup, we colonised four mice with two commensal strains of E. coli (marked with two fluorescent markers: yellow fluorescent protein [YFP] and cyan fluorescent protein [CFP]; both streptomycin-resistant) and followed their dynamics for 190 days (approximately 3,600 generations, assuming 19 generations/day; Fig 1A) [38-40]. Both strains carry mutations that we previously showed to be adaptive in the mouse gut [38,39]. Mice 1 and 2 were co-colonised with a YFP-labelled strain with a 1-bp insertion in gatC and a CFP-labelled strain with the same gat $C$ mutation and two other mutations: an IS2 insertion between $y j j P$ and $y j j Q$ and a $4 \mathrm{bp}$ insertion between $y j j Y$ and $y j t D$. Mice 3 and 4 were co-colonised with the same genetic backgrounds, with the single mutant expressing CFP and the triple mutant expressing YFP. All mice were co-colonised with the triple mutant at a lower starting frequency (10\%), as this carries more beneficial mutations and has a higher fitness under the 7-day streptomycin treatment (S1 Fig). Throughout the entire experiment, both strains coexisted in all mice, and E. coli generally maintained a population size of $>10^{7}$ colony-forming units (CFU)/g of faeces (S1 Fig), i.e., the typical size of its ecological niche in this host [41].

As mutation rate is a key evolutionary parameter that can evolve, both in vitro and in vivo (e.g., $[8,17,23-25,30])$, we screened a sample of clones from each mouse ( $n \approx 90$ per mouse) for potential changes in mutation rate (Fig 1B). In one of the four mice, we detected clones with increased mutation rate. To understand the magnitude and dynamics of the mutation rate increase in this host, we performed Luria-Delbrück fluctuation assays for several clones sampled at days $45,136,148$, and 190 and measured their mutation rate towards nalidixic acid resistance ( $n=10$ independent growths per clone; Fig 1C). At day 45, none of the clones exhibited a mutation rate (towards resistance) significantly different from that of the ancestral $\left(5 \times 10^{-10}\right.$; per cell per generation; $95 \%$ CIs for all clones overlap with those of the ancestral). However, from day 136 onwards, mutation rate varied by three orders of magnitude, ranging between $4.7 \times 10^{-10}$ and $6 \times 10^{-7} /$ cell/generation. Similar levels of mutation rate variation were observed at days 148 and 190 (range at day $148: 3.1 \times 10^{-10}$ to $2.8 \times 10^{-7}$; day 190: $1.2 \times 10^{-10}$ to $4.5 \times 10^{-7}$ per cell per generation). This indicates that multiple mutators emerged either simultaneously or sequentially and that nonmutator clones coexisted with both strong hypermutators (mutation rates up to 1,200-fold higher than the ancestral) and with intermediate hypermutators (10- to 100 -fold increases in mutation rate). Coexistence between clones differing in mutation rate by less than 70 -fold has been previously observed [42-45]. However, to the best of our knowledge, our results represent the first observation of long-term coexistence 


\section{A. Long-term experimental evolution of E. coli in the mouse gut}

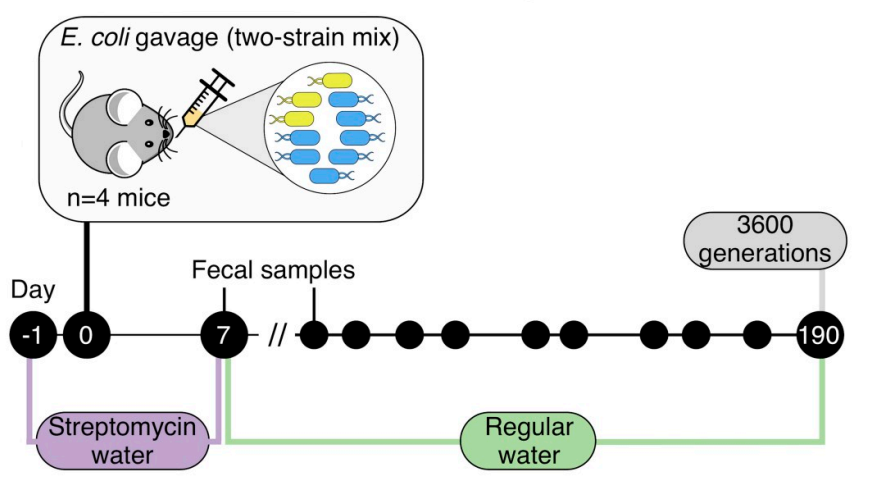

B Ancestral

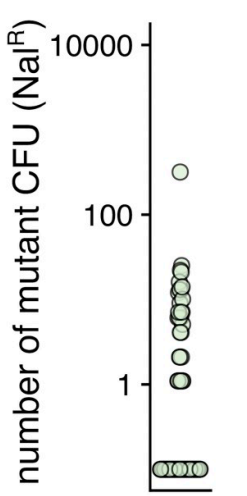

Clones sampled at 190 days post-colonization

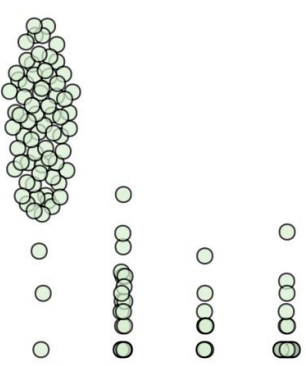

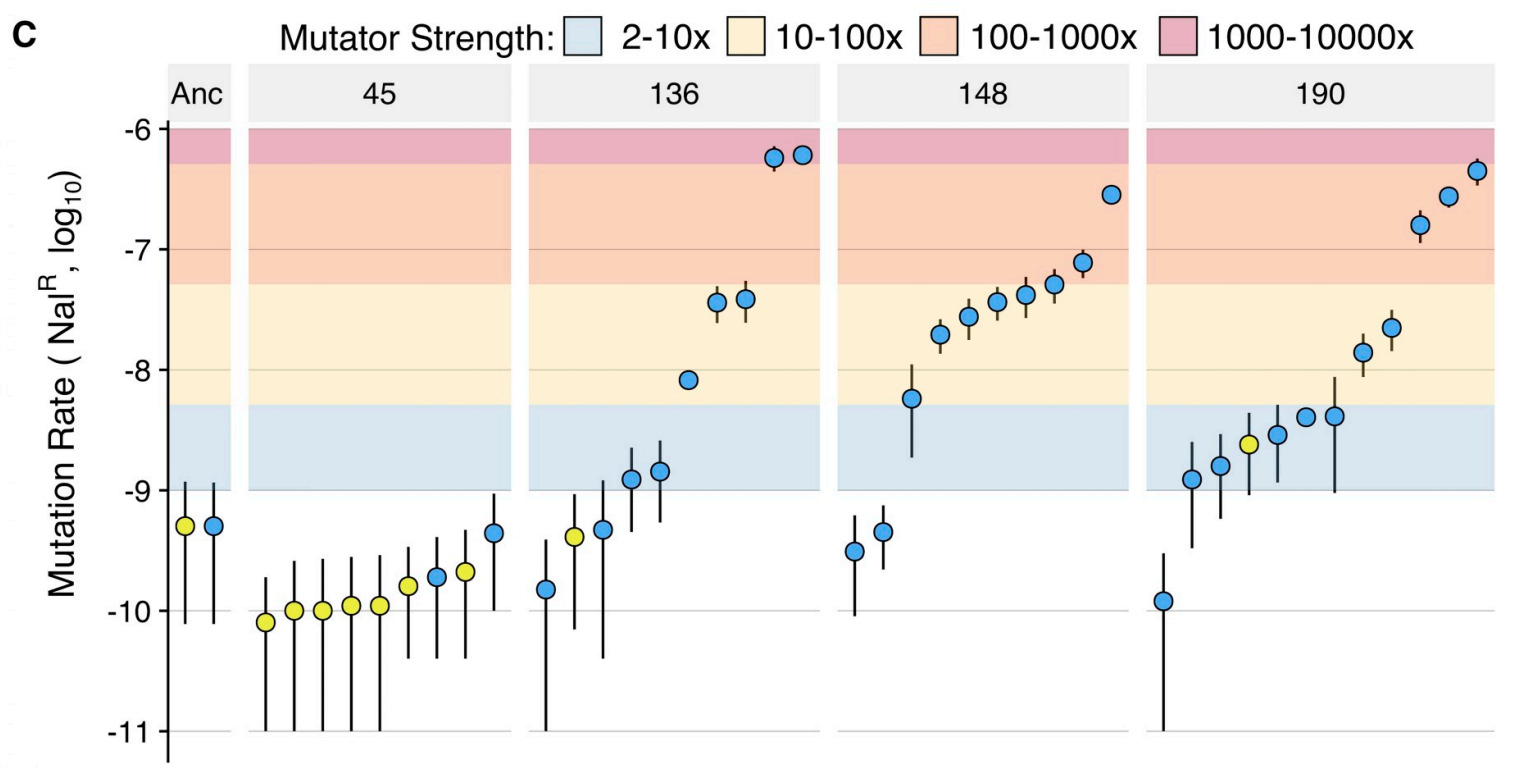

Fig 1. Multiple clones with variable mutation rates (up to 1,000-fold higher than ancestral) emerge and coexist during long-term adaptation to the mouse gut. (A) Scheme of the experimental design used to follow the long-term adaptation of $E$. coli to the mouse gut. (B) Screen for mutation rate variation shows that mutators emerged in mouse 1. A total of 83 to 90 clones, isolated from each mouse at day 190 post colonisation, were grown overnight and plated in nalidixic acid. The number of resistant colonies obtained for each clone is shown. The same procedure was carried for 96 replicates of the ancestral clones. (C) Large-scale variation for mutation rate emerges in mouse 1. Mutation rates (towards $\mathrm{Nal}^{\mathrm{R}}$ ), measured through fluctuation tests, for multiple clones randomly isolated at different time points from mouse 1. Each point corresponds to one clone. Error bars represent 95\% CIs of 10 independent growths (nonoverlapping bars indicate significant differences). Blue and yellow points correspond to clones from the CFP and YFP backgrounds. Coloured areas indicate the mutation rate fold-change of a particular evolved clone relative to the ancestral (i.e., mutator strength). CFP, cyan fluorescent protein; $\mathrm{CFU}$, colony-forming units; $\mathrm{Nal}^{\mathrm{R}}$, nalidixic acid resistance; YFP, yellow fluorescent protein.

https://doi.org/10.1371/journal.pbio.3000617.g001

( $>1,000$ generations, i.e., $>54$ days) of clones differing in mutation rate by around 1,000-fold in the gut.

These observations raised two main questions: What is the genetic basis for the observed mutation rate increase? Under what evolutionary conditions could we expect the emergence of 1,000-fold mutators and the long-term maintenance of mutation rate polymorphism? 


\section{Mutations in the proofreading and catalytic subunits of DNA polymerase III cause hypermutability and improve mutator fitness, respectively}

To determine the cause of the observed mutation rate variation, we carried out whole-genome sequencing of 18 clones, 13 mutators and 5 nonmutators, isolated from mouse 1 (all CFP), between day 136 and 190 post colonisation (Fig 2A). Mutator and nonmutator clones accumulated 164 to 658 and 4 to 5 mutations (respectively) and followed independent evolutionary paths (Fig 2A). Observing 4 to 5 mutations in nonmutator clones after 6 months is not unexpected, given that the nonmutators are competing with mutator clones and that previous experiments showed 1 to 4 adaptive mutations in nonmutator clones after 1 month of colonisation of the mouse gut (approximately 450 generations [38,39]). All mutator clones shared 45 mutations, none of which were present in the isolates that maintained the ancestral mutation rate (Fig 2A and S1 Table). Among these, we identified a single nonsynonymous mutation in the gene $d n a Q$, which could increase mutation rate by 1,000 -fold. $d n a Q$ encodes the proofreading $(\varepsilon)$ subunit of DNA polymerase III [46], the main DNA polymerase in E. coli. Following their divergence, mutators accumulated mutations in several other genes that may affect mutation rate (S2 Fig; none of these genes were mutated in the nonmutators; S2 Table). Strikingly, these included several mutations in two different subunits of DNA polymerase III: the catalytic $(\alpha)$ subunit (encoded by $d n a E$; mutated in 11 clones) [47] and the $\theta$ subunit (holE; two clones) [48]. The $\alpha, \varepsilon$, and $\theta$ subunits physically interact to form the core of DNA polymerase III (Fig 2B) [20,49]. Nonsynonymous mutations in $d n a Q$ were previously shown to cause mutation rate increases up to $>5,000$-fold [19]. Mutations in $d n a E$ and to a smaller extent in holE were also shown to have antimutator effects when linked to $d n a Q$ mutations $[50,51]$. This led us to hypothesise that a mutation in $d n a Q$ first raised the mutation rate, which was later reduced by subsequent mutations in $d n a E$, thus leading to the observed mutation rate polymorphism. To query whether the $d n a Q$ mutation caused the hypermutator phenotype, and whether $d n a E$ could act as a modifier, we engineered single and double mutants with the $d n a \mathrm{Q}$ mutation (L145P; CTC $\rightarrow$ C $\underline{C}$ ) and a mutation in $d n a E$ (T771S; $\underline{\mathrm{ACG}} \rightarrow$ TCG) and measured their mutation rates. We generated approximately 20 clones carrying the $\overline{\mathrm{D} n a Q} \mathrm{Q}^{\mathrm{L} 145 \mathrm{P}}$ allele, as $d n a \mathrm{Q}$ hypermutators can exhibit high mutation rate variation, possibly because of the rapid emergence of suppressors [42,51]. The $d n a Q$ clones indeed showed a very high mutation rate, reaching 3,000-fold increases for both nalidixic acid and rifampicin (Fig 2C). The distribution of mutation rate estimates is bimodal, with a group of clones showing approximately 200 -fold increase in mutation rate, whereas another shows an increase of approximately 1,000-fold (none of the 95\% CIs overlap with the ancestral; S3 Table). Given the observed mutation rate increase of about 1,000-fold for clones isolated from mice and that it is possible for mutation rate variation to emerge during the engineering process (because of suppressors; see [51]), we suggest that the $d n a Q$ mutation is responsible for the approximately 1,000 -fold mutation rate increase.

Contrary to our hypothesis, the DnaE $\mathrm{E}^{\mathrm{T} 771 \mathrm{~S}}$ mutation did not cause any significant change in the mutation rate on the ancestral or the DnaQ ${ }^{\mathrm{L} 145 \mathrm{P}}$ genetic backgrounds (Figs $2 \mathrm{C}$ and S3). This suggests that the $d n a E$ mutation did not act to decrease mutation rate. However, the independent occurrence of different alleles in the $\alpha$ subunit of DNA polymerase III (Figs 2B and S2) strongly suggests that mutations in $d n a E$ could be beneficial. Consistent with this hypothesis, we observed that DnaE ${ }^{\mathrm{T} 771 \mathrm{~S}}$ strongly improves growth of the DnaQ ${ }^{\mathrm{L} 145 \mathrm{P}}$ mutant (Figs 2D and S4). Remarkably, the DnaQ ${ }^{\mathrm{L} 145 \mathrm{P}}$ mutation is lethal in minimal media with a single carbon source (either glucose or sorbitol). Its null fitness can be rescued by complementing minimal media with amino acids or in lysogeny broth (LB), which allows the DnaQ ${ }^{\mathrm{L} 145 \mathrm{P}}$ mutant clones 
A

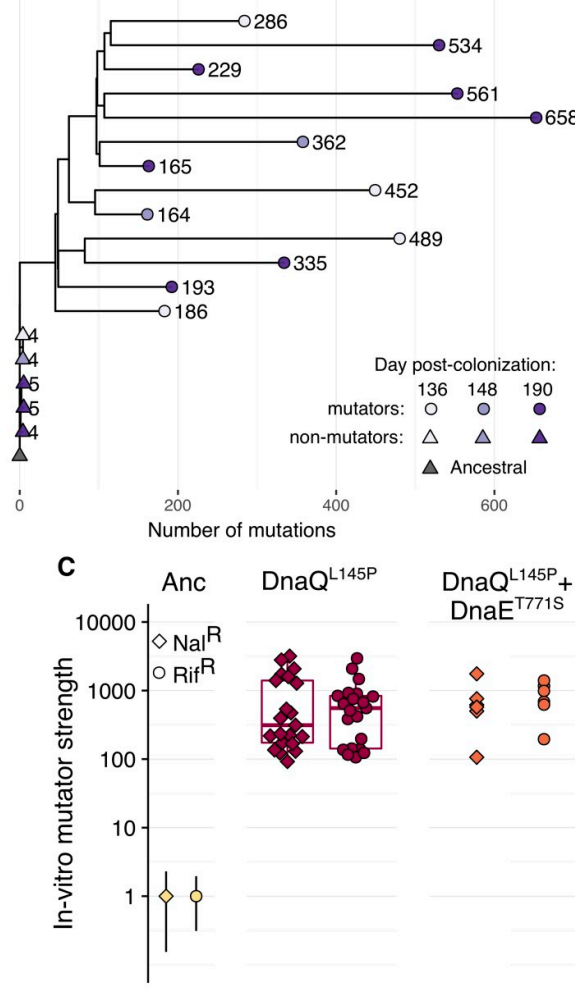

B core DNA pol. III structure

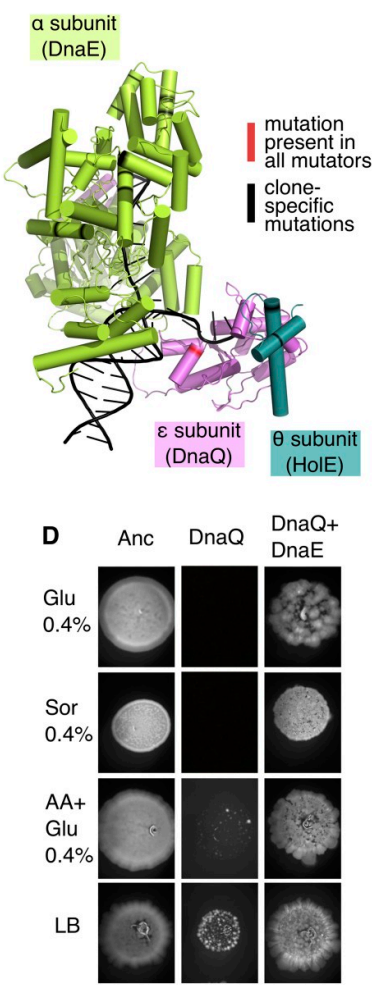

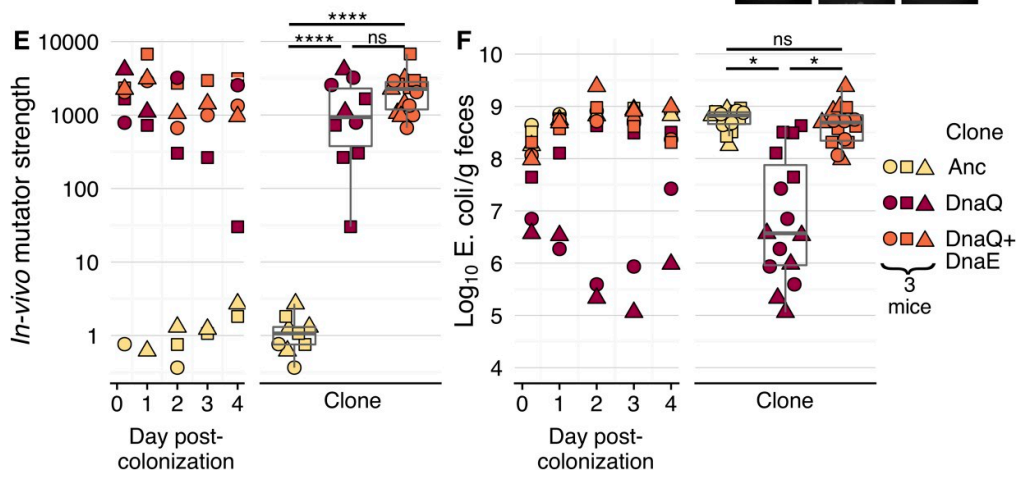

Fig 2. Mutations in DNA polymerase III both drive mutation rate increase by $1,000-$ fold (DnaQ ${ }^{\mathrm{L145P}} ; \varepsilon$ subunit) and rescue fitness of hypermutator clones $\left(\mathrm{DnaE}^{\mathrm{T} 771 \mathrm{~s}} ; \boldsymbol{\alpha}\right.$ subunit). (A) Phylogenetic tree of 18 clones from mouse 1 and the ancestral strain generated from whole-genome sequence data. Mutator and nonmutators have independent evolutionary histories, with all mutators sharing 45 mutations. Branch lengths and tip labels indicate the number of accumulated mutations. Tip point colours indicate the day post colonisation at which different clones were isolated. (B) Structure of the core DNA polymerase ("pol.") III (PDB ID: 5M1S), with each subunit in a different colour and nonsynonymous mutations highlighted in red or black. (C) In vitro fluctuation tests were performed for the ancestral clone ("Anc"), the single mutant DnaQ ${ }^{\mathrm{L} 145 \mathrm{P}}$, and the double mutant $\mathrm{DnaQ}^{\mathrm{L} 145 \mathrm{P}}+\mathrm{DnaE}^{\mathrm{T} 771 \mathrm{~S}}$ (21 and 5 independent clones were tested for each mutant, respectively). Each point shows the mutation rate fold-change of a particular evolved clone relative to the ancestral (i.e., in vitro mutator strength; 10 replicates per clone for ancestral and double mutant; 5 replicates per clone for DnaQ ${ }^{\mathrm{L} 145 \mathrm{P}}$ ). For ease of visualisation, $95 \% \mathrm{CIs}$ are only shown for the ancestral, but none of the 95\% CIs of either mutant is overlapping with those of the ancestral (see S3 Fig for the mutation rate of $\mathrm{DnaE}^{\mathrm{T771S}}$ and $\mathrm{S} 3 \mathrm{Table}$ for mutation rates and $95 \% \mathrm{CIs}$ ). (D) In vitro phenotypic growth capacity of the ancestral clone, the single mutant $\mathrm{DnaQ}{ }^{\mathrm{L} 145 \mathrm{P}}$, and the double mutant $\mathrm{DnaQ}^{\mathrm{L} 145 \mathrm{P}}+\mathrm{DnaE}^{\mathrm{T} 771 \mathrm{~S}}$ (spot assay; all inocula had the same $\mathrm{OD}_{600 \mathrm{~nm}}$; similar results were obtained for an extended number of clones, see $\mathrm{S} 4 \mathrm{Fig}$ ). (E) In vivo dynamics and summary box plots of the mutation frequency fold-change relative to the average mutation frequency obtained for the ancestral strain (i.e., in vivo mutator strength; similar results were obtained when measuring mutation frequency towards $\mathrm{Nal}^{\mathrm{R}}$; S6 Fig); (F) In vivo dynamics and summary boxplots of $E$. coli CFU per gram of faeces. AA, amino acids; $\mathrm{CFU}$, colony-forming units; Glu, glucose; $\mathrm{LB}$, lysogeny broth; $\mathrm{Nal}^{\mathrm{R}}$, nalidixic acid resistance; ns, not significant; $\mathrm{OD}_{600 \mathrm{~nm}}$, optical density at $600 \mathrm{~nm}$; PDB, Protein Data Bank; Rif ${ }^{\mathrm{R}}$, rifampicin resistance; Sor, sorbitol.

https://doi.org/10.1371/journal.pbio.3000617.g002 
to achieve slow but visible growth (Fig 2D). Furthermore, the double mutant (DnaQ ${ }^{\mathrm{L} 145 \mathrm{P}}+$ $\mathrm{DnaE}^{\mathrm{T771S}}$ ) grows to levels similar to the ancestral strain across all media.

As mutation rate and spectrum can depend on the environment (e.g., [52]), we sought to determine the mutation rate of the $d n a Q$ and $d n a Q+d n a E$ mutant strains in vivo. The classical Luria-Delbrück in vitro assay, designed to avoid selection, is inappropriate for estimating the mutation rate in the gut. However, we can use Haldane's mutation-selection balance principle to estimate the in vivo mutation rate of these clones. We colonised mice with the ancestral strain, the $d n a Q$ mutant, and the double $d n a Q+d n a E$ mutant and measured the equilibrium frequency of rifampicin-resistant $\left(\mathrm{Rif}^{\mathrm{R}}\right)$ clones ( $n=3$ mice for each genetic background). Under mutation-selection balance, the frequency of resistant mutants is directly proportional to the mutation rate [53]. Thus, the ratio of the $\mathrm{Rif}^{\mathrm{R}}$ mutation frequency in the mutants, relative to the ancestral strain, provides an estimate of the fold-change in mutation rate (i.e., mutator strength). The mutation frequency (towards resistance) of either the single $d n a Q$ mutant or the double $d n a Q+d n a E$ mutant is about 1,000-fold higher than observed for the ancestral strain but not significantly different between mutants (Fig 2E, see also S5 Fig for comparison between mutation frequency in vivo and in an in vitro propagation; linear mixed model followed by Tukey test: clone effect: $\chi_{2}^{2}=31.9, p<0.0001$; ancestral versus $d n a Q: t=-12.8$, $d f=6, p<0.0001$; ancestral versus $d n a Q+d n a E: t=-14.9, d f=6, p<0.0001$; dna $Q$ versus $d n a Q+d n a E: t=-1.5, d f=6, p=0.37)$. Similar results were obtained when using nalidixic acid as the selection marker (S6 Fig), which targets a different region of the genome [54,55]. When comparing the niche size that each strain occupies in the mouse gut, we find that the average abundance of the $d n a Q$ mutant $\left(10^{6}\right.$ per gram of faeces) is significantly lower than that of the ancestral strain $\left(>10^{8}\right.$ per gram of faeces; linear mixed model followed by Tukey test: clone effect: $\chi_{2}^{2}=10.2, p=0.006$; ancestral versus $d n a Q: t=-3.7, d f=6, p=0.022$ ). Remarkably, the fitness defect of $d n a Q$ is fully rescued by the $d n a E$ mutation (Fig $2 \mathrm{~F}$ ), as the bacterial loads for the double mutant are similar to those for the ancestral strain $\left(>10^{8}\right.$ per gram of faeces; ancestral versus dnaQ+dnaE: $t=0.2, d f=6, p=0.97$; dnaQ versus dnaQ+dnaE: $t=-3.5, d f=6$, $p=0.029$ ). These results are similar to those observed in vitro, when the strains grow in different nutrient sources. Taken together, our results establish that the $d n a Q$ mutation massively increases mutation rate both in vitro and in vivo, albeit at a fitness cost. This cost can be recovered by a beneficial mutation in $d n a E$, without strong effects on mutation rate. The causes of mutation rate polymorphism are likely to be complex, as most mutator clones accumulated mutations in $\geq 3$ genes that can affect mutation rates (e.g., a single clone can have mutations in $d n a Q, d n a E$, recF, katE, and $s o d B$; S2 Fig). Interestingly, although clones with 1,000-fold increase in mutation rate (unknown genetic basis) have been observed in humans in the context of infection $[17,56]$, to our knowledge, $d n a Q$ mutators have not yet been reported in natural populations.

\section{Survival of hypermutators indicates that deleterious mutations are of small effect}

Clonal populations with very high genomic mutation rates can quickly accumulate many deleterious mutations and experience severe impediments on fixing adaptive mutations $[57,58]$. Because of the accumulating mutational load, such strong mutators may continuously decay in fitness (sensu Muller's ratchet $[59,60]$ ) in a potentially inescapable path towards extinction $[61,62]$. However, we observe clones with 1,000-fold increase in mutation rate that coexist with nonmutators and intermediate mutators for $>1,000$ generations (Fig $1 \mathrm{C}$ ) and a high variation in the number of accumulated mutations across mutator clones (Fig 2A). Furthermore, E. coli loads in mouse 1 are approximately 10-fold lower than in any other mouse (S1 Fig), 
specifically after the detection of mutators, which should lead to an even faster accumulation of mutational load. These observations raise two questions: Under which conditions can such strong mutators avoid a catastrophic fitness decline, caused by the accumulation of deleterious mutations $[59,60]$ ? And what conditions allow for coexistence between mutator and nonmutator clones?

To explore theoretical scenarios under which the above observations could be expected, we simulated clonal populations evolving under many mutation rate values and considered different assumptions for the fitness effects of mutations. We started by simulating the simplest model, assuming that all mutations are deleterious with a fixed and constant effect and ignoring neutral, lethal, compensatory, or beneficial mutations. We simulated populations of $10^{6}$ individuals (similar to E. coli's population size in the gut; S1 Fig) under many combinations of mutation rate ( $U$; per genome per generation) and the fitness effects of deleterious mutations $\left(s_{d}\right.$; per generation). We then quantified mean fitness and the number of accumulated mutations after a time similar to that over which mutator maintenance was observed $(1,000$ and 2,000 generations). First, we consider fixed $s_{d}$ values and show that for clones with a wild-type mutation rate $(U=0.001$ per genome per generation), fitness remains at approximately 1 independently of $s_{d}$ (Figs 3 Ai and S7). However, when $U>0.1$, fitness depends on $s_{d}$. At $s_{d}$ values between 0.001 and 0.01 , typical estimates from in vitro MA experiments with bacteria (which assume a constant $s_{d}$ or estimate an average $s_{d}$ ) [31-35], the mean fitness of lineages with $U>0.5$ declines to $<0.68$ within 1,000 generations (Fig 3Ai) and is even lower after 2,000 generations (see S7 Fig and S4 Table for a broader parameter space at 1,000 and 2,000 generations). Therefore the long-term persistence of such hypermutators is unlikely. In contrast, mean fitness remains high when $s_{d} \leq 0.0001$, across mutation rates spanning three orders of magnitude, and the level of variation for the number of accumulated mutations in mutator populations is similar to what we observe in the evolution experiment (indicated by the boxed numbers in Fig $3 \mathrm{Ai}$ ). Next, we assumed a fraction $f_{\text {neut }}$ of neutral and $1-f_{\text {neut }}$ of deleterious mutations with a fixed effect (Fig 3Aii and 3Aiii), since many mutations are expected to be neutral. Neutral mutations allow hypermutators to maintain high fitness across a wider range of $s_{d}$ values. With $f_{\text {neut }}=0.9$ (Fig 3Aiii), populations with high $U$ may avoid extinction, for thousands of generations, even under $s_{d}=0.01$. However, irrespectively of $f_{\text {neut }}$, when $s_{d} \leq$ 0.0001 mutator populations can maintain fitness at approximately 1 despite accumulating hundreds of mutations (Figs 3A and S7). These results suggest that coexistence between nonmutators and strong hypermutators is possible when $s_{d} \leq 0.0001$ regardless of $f_{\text {neut }}$, or $f_{\text {neut }}=$ 0.9 , irrespectively of $s_{d}$. Simulations of populations with a 50:50 mixture of mutators (1,000-fold increase in mutation rate) and nonmutators competing for 1,000 generations (the minimum time over which mutators and nonmutators coexisted; Fig 1C) predict coexistence between mutator and nonmutator clones when $s_{d} \leq 0.0001$ (Fig 3C) but not when $s_{d}$ is 0.001 or 0.01 . We note that lethal mutations are ignored in these simulations. These can be an important source of mutational load that could impair mutator persistence. Under a more realistic scenario, spontaneous mutations were assumed to follow an exponential distribution of deleterious effects (with mean effect $s_{d}$ per generation). Under such conditions (Fig 3Bi and $3 \mathrm{Di}$ ), we obtain results similar to those when $s_{d}$ has a fixed and constant effect (Fig 3Ai and 3Ci).

Next, we evaluated the impact of beneficial mutations. In vitro estimates suggest a fraction of beneficial mutations at $1 \%$ in $E$. coli $\left(U_{b}=10^{-5}\right.$ per genome per generation) with average effect $s_{b}=0.01$ (per generation; [63]). Moreover, strong-effect beneficial mutations have been inferred in the mouse gut $\left(s_{b}\right.$ between 0.02 and 0.12 per generation; [39,64]). Thus, we assumed different proportions of beneficial mutations ( $1 \%$ or $10 \%)$, with the fitness effects of deleterious and beneficial mutations drawn from two independent exponential distributions (with 

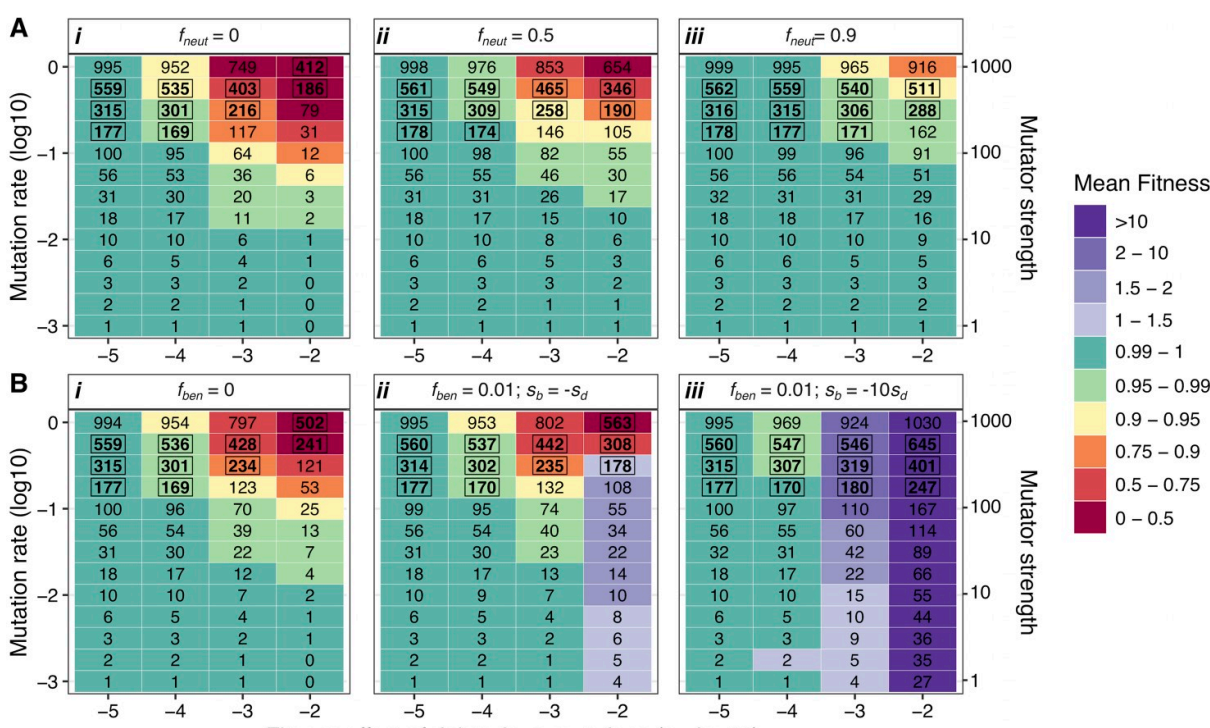

$0.99-1$

$0.95-0.99$

$0.9-0.95$

$0.75-0.9$

$-100$

$0.75-0.9$

$0-0.5$
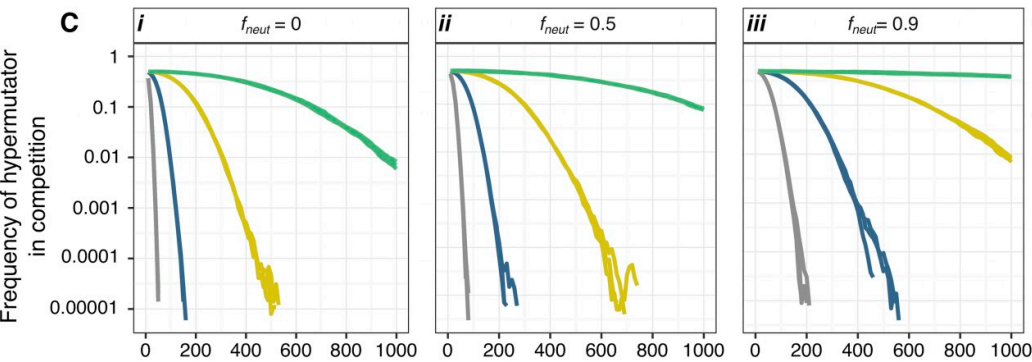

Fitness effect of deleterious

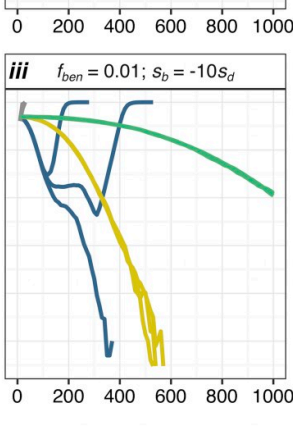

mutations $\left(s_{d}\right)$
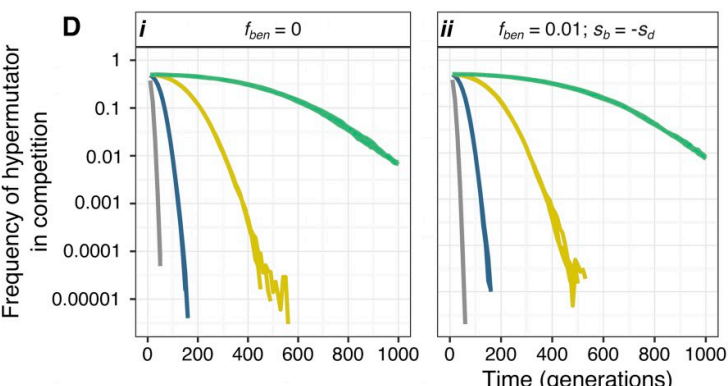

$-0.01$

$-0.001$

-0.0001
$-\quad-0.00001$

Fig 3. Strong, intermediate, and weak mutators can maintain high fitness and coexist if deleterious mutations have very weak effects. (A-B) Simulations with a monoclonal population. Numbers indicate the mean number of mutations, and the colour gradient indicates mean fitness after 1,000 generations (initial fitness is 1). Numbers in bold and inside a box indicate the parameter space for which there is a fit between the observed and the simulated number of mutations ( $n=10$ simulations for panel Ai and $n=3$ simulations for all others; see S4 and S5 Tables for the mean and standard deviation of fitness and the number of mutations). (C-D) Temporal dynamics of competition between two clones differing in mutation rate by 1,000 -fold $(U=1$ and $U=0.001$ per genome per generation; equivalent to the DnaQ ${ }^{\mathrm{L} 145 \mathrm{P}}$ mutant and the ancestral, respectively; dynamics of 3 simulations per parameter combination are shown). All populations had $10^{6}$ individuals, which evolved under different distributions of the fitness effect of mutations. (A and C) Mutations were either all deleterious with a fixed fitness effect (panel i) or neutral, with a fraction of neutral mutations $\left(f_{\text {neut }}\right)$ of $50 \%$ or $90 \%$ (panels ii-iii). (B and D) Either mutations were all deleterious (panel i), or a $1 \%$ fraction $\left(f_{\text {ben }}\right)$ was beneficial. The fitness effects of deleterious and beneficial mutations were drawn from two independent exponential distributions, with mean effects being indicated in each panel (see S7, S8, S9, and S10 Figs for a broader exploration of the parameter space and S11, S12, S13, S14, and S15 Figs for $N=10^{5}$ ).

https://doi.org/10.1371/journal.pbio.3000617.g003

mean effect $s_{d}$ or $s_{b}$ per generation, respectively), which may or may not be symmetrical (we tested the following mean $s_{b}$ : $-s_{d},-10 s_{d}, 0.01$, and 0.05 ). The occurrence of strong-effect beneficial mutations $\left(s_{b} \geq 0.01\right)$ leads to high variation in mean fitness under different $U$ values, for large $s_{d}$ (Figs 3Bii and 3Biii and S9 and S5 Table). Consistent with these results, coexistence 
between clones differing in mutation rate by 1,000 -fold is short lived ( $<500$ generations) across all $s_{d}$ values when $s_{b} \geq 0.01$ (Figs 3Dii and 3Diii and S10). Conversely, the number of observed mutations (Fig $2 \mathrm{~A}$ ) and the coexistence of clones with mutation rates differing by three orders of magnitude (for 1,000 generations) can be expected when $s_{b}<0.0001$, but only if $s_{d} \leq 0.00001$ (Figs 3Dii and 3Diii and S10; similar results were obtained with $10 \%$ of beneficial mutations).

The theoretical expectations, constrained by the experimental observations (in vivo accumulation of mutations and long-term maintenance of mutation rate polymorphism; Figs 1C, $2 \mathrm{~A}$, and 3), are consistent with low fitness effects of deleterious mutations. The same conclusion is reached under different distributions of the fitness effects of mutations and at two reasonable population sizes (see S11, S12, S13, S14, and S15 Figs for simulations under a population size of $10^{5}$ ). We note these simulations ignore temporal variation in the distribution of fitness effects of beneficial and deleterious mutations, which could affect the inferences. Thus, within the simplifying assumptions made in the simulations, the fitness effect of deleterious mutations in the mouse gut appears to be lower than what was previously inferred in vitro in rich media for $E$. coli from classical MA experiments $\left(\mathrm{s}_{\mathrm{d}} \approx 0.01\right.$ to 0.03 , assuming a fixed effect; [31,32]) and closer to recent single-cell estimates ( $s_{\mathrm{d}} \approx 0.003$; [35]).

To complement the inference from the simulations, we sought to experimentally compare the deleterious mutational load in vivo and in vitro. We tested clones with a large number of deleterious mutations, acquired under conditions of minimal selection, for their mutational load both in vitro and in the mouse gut. We used the double mutant $\mathrm{DnaQ}^{\mathrm{L} 145 \mathrm{P}}+\mathrm{DnaE}^{\mathrm{T} 771 \mathrm{~S}}$, which should acquire about 1 new mutation per generation, to seed a short MA experiment. Two bacterial lineages were streaked on LB agar plates and allowed to grow from a single cell to $10^{8}$ cells, every 24 hours (i.e., approximately 25 generations per day). After 4 passages, we isolated clones (carrying a CFP marker) from the two independent MA lines (each line should have accumulated about 100 mutations) and competed these against the ancestral $\mathrm{DnaQ}^{\mathrm{L} 145 \mathrm{P}}+\mathrm{DnaE}^{\mathrm{T} 771 \mathrm{~S}}$ (carrying a YFP marker). As expected, fitness declined in all competitions (Fig 4A), but remarkably, the decline was much steeper in vitro than in vivo (Fig 4A and $4 \mathrm{~B})$. These data show that the same mutator lineage, loaded with deleterious mutations, will typically exhibit a lower mutational load in the gut than in rich LB media. Interestingly, one of the MA lines went extinct in vitro (it could not be detected in 3 of 4 replicates) but not in vivo. The combination of results indicates that levels of fitness decline are 10- and 4-fold lower for line 1 and 2 (respectively) in vivo than in vitro (linear mixed model: $\chi^{2}{ }_{1}=21.8, p<0.0001$ ). This difference between in vitro and in vivo is likely an underestimate, given that natural selection is not completely absent in MA experiments [34] and because we carried the MA and the in vitro competitions in LB.

The above experimental results concur with the inference from the simulations that the fitness effect of deleterious mutations is lower in vivo than in vitro. The estimated effects of deleterious, but nonlethal, mutations are compatible with the observed dynamics of mutators in a natural environment and close to typical estimates of the fitness effect of deleterious mutations obtained from analysis of natural polymorphism (e.g., for Drosophila, $s_{d} \approx 10^{-5}-10^{-4}$ when estimated from natural polymorphism; [65,66]). Moreover, despite the gap in knowledge regarding the causes of polymorphism in commensal bacteria [67], the high level indicates that deleterious mutations should typically have small effects [68,69]. Remarkably, by analysing polymorphism patterns in species of the human gut microbiome (including E. coli), Garud and colleagues [5] recently estimated $s_{d} / \mu=10^{5}$ ( $\mu$ represents the mutation rate per site per generation). Given that our data indicate that the spontaneous mutation rate is similar in vitro and in vivo, if we take the mutation rate per site per generation estimated for E. coli from in 

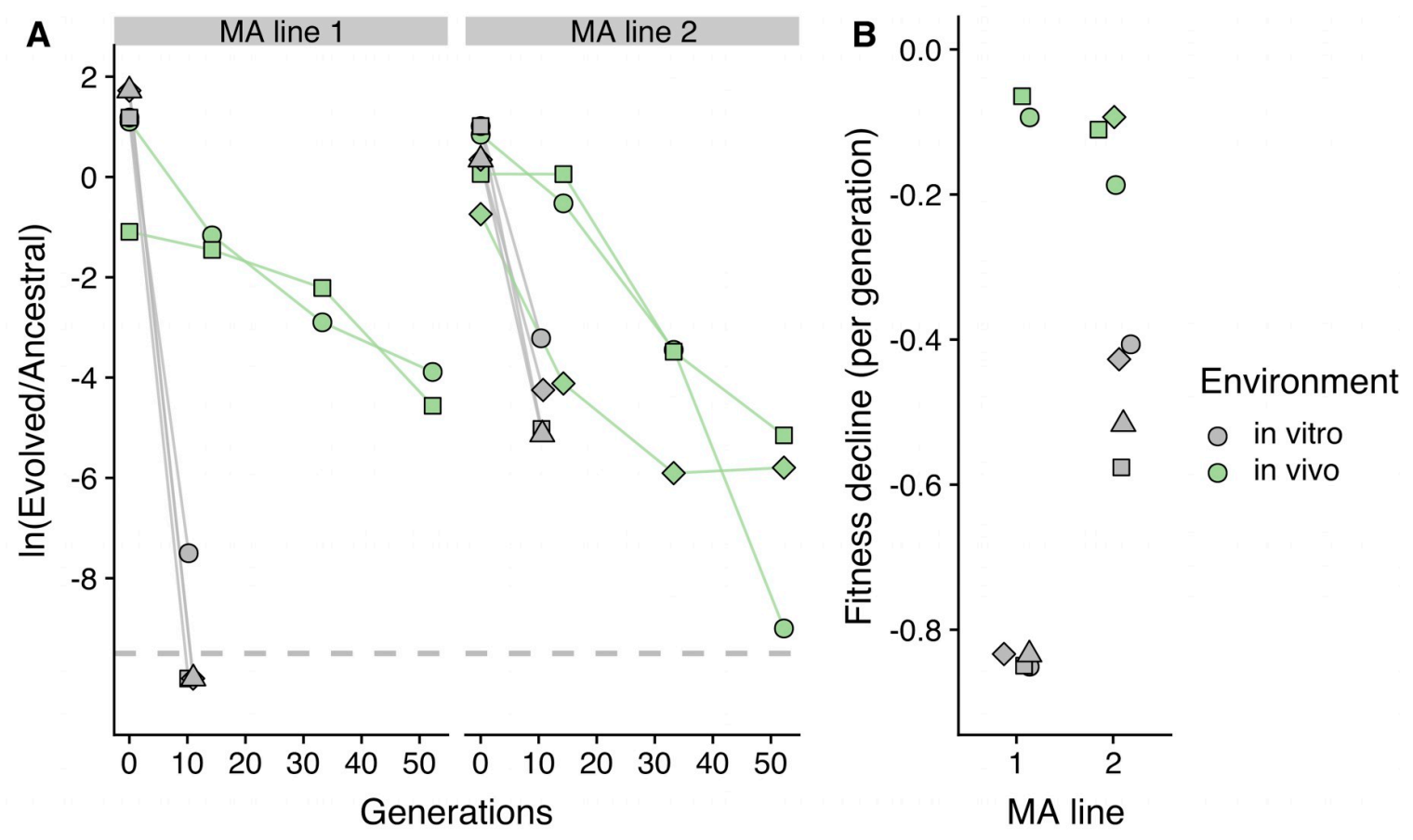

Fig 4. Competitive fitness assays demonstrate that the fitness effects of mutations are lower in the gut than in vitro. A short $M A$ experiment was performed, starting from a DnaQ ${ }^{\mathrm{L} 145 \mathrm{P}}+\mathrm{DnaE}^{\mathrm{T} 771 \mathrm{~S}}$ clone. Two lines were propagated for 4 passages (approximately 100 generations). These MA lines were propagated in conditions of nearly neutral evolution to accumulate many deleterious mutations. The evolved lines were then competed against the ancestral DnaQ ${ }^{\mathrm{L} 145 \mathrm{P}}+\mathrm{DnaE}^{\mathrm{T} 771 \mathrm{~S}}$ clone, either in vitro (for 1 day; approximately 10 generations) or in vivo, in the mouse gut (for 3 days; approximately 50 generations). (A) Temporal dynamics of the ratio between each evolved line and the ancestral, during 50 generations of competition. The natural logarithm of the ratio of the population sizes of the two genotypes is shown. Dashed line indicates the detection limit for colonies of the evolved lines. (B) Fitness decline for the two MA lines, when competing against the ancestral strain, both in vitro and in vivo. Fitness decline is estimated as the slope of the natural logarithm of the ratio between each evolved line and the ancestral. In 3 of the 4 in vitro replicates for line 1, we could not detect any colonies of the evolved clone. To estimate a fitness decline for these replicates, we assumed 1 colony for the evolved clone. We note that the values shown in Fig 4B are the fitness declines per generation of clones that have accumulated an unknown number of mutations and thus cannot be directly compared with the estimates of the fitness effect per deleterious mutation inferred in the simulations. MA, mutation accumulation.

https://doi.org/10.1371/journal.pbio.3000617.g004

vitro MA experiments $\left(\mu=10^{-10}[70]\right)$, we obtain an $s_{d}$ value of approximately $10^{-5}$, which is consistent with our results.

\section{Adaptive mutations target metabolism and stress resistance}

Whereas weak-effect deleterious mutations can explain the observed molecular evolution patterns, they cannot explain mutator invasion. In in vitro evolution experiments, mutators have been observed to invade through hitchhiking with beneficial mutations (i.e., mutations causing the mutator phenotype increase in frequency because these occur in the same genotype as a beneficial mutation; [24,71,72]). Given the fitness defect of the $d n a Q$ mutation described above (in vitro and in vivo), and because beneficial mutations can rapidly emerge during adaptation to the mouse gut [38-40,73], we propose that hitchhiking is also the most likely route driving the observed increase in mutation rate. Thus, we sought to find signatures of adaptation in the mutator clones. First, we computed the ratio of the rates of nonsynonymous to synonymous mutations, $\mathrm{dN} / \mathrm{dS}$, to detect whether there was evidence of either purifying ( $\mathrm{dN} /$ $\mathrm{dS}<1)$ or positive selection $(\mathrm{dN} / \mathrm{dS}>1)$ [74]. Consistent with the above modelling inference, in which a large number of very slightly deleterious mutations, or even neutral mutations, accumulated in the mutators, $\mathrm{dN} / \mathrm{dS}$ showed a weak signal of purifying selection $(\mathrm{dN} / \mathrm{dS}<1$ 
in 3/13 clones; $p<0.05$; S16 Fig). Furthermore, no signs of positive selection could be detected using this statistic (a well-known difficulty for clonal populations).

Because of the power of our experimental design, in which the same colonising lineages evolve independently in genetically identical animals under the same diet, one can identify beneficial mutations through mutational parallelism. We isolated 3 to 4 clones per mouse (from the remaining 3 mice) 190 days post colonisation and carried out whole-genome sequencing. We then identified genes or operons that had been repeatedly mutated across hosts (i.e., parallelism; S17 Fig) as likely targets of natural selection. Using this approach, we identified 12 targets, 8 of which were also mutated in the mutator clones. Of these, $p t s P, f r l R$, and $d g o R$ were mutated in all mutators (i.e., in the mutator common ancestor), whereas 5 other targets were mutated in at least two mutator clones (Fig 5).

Most parallel targets in the mutators have a metabolic function, and some have been previously observed in other mouse gut colonisation experiments. Specifically, whereas $p t s P$ is a novel target of adaptation, $f r l R$ and $d g o R$ have been previously identified as targets of adaptation to the gut of streptomycin-treated immunocompromised or wild-type hosts, respectively $[40,73]$. frl $R$ and $d g o R$ are negative regulators of the $f r l$ and $d g o$ operons [73,75,76], which are involved in carbon metabolism, namely of fructoselysine and galactonate (respectively). ptsP encodes a phosphoenolpyruvate-protein phosphotransferase, which is thought to be involved in the response to nitrogen availability and to several stresses (e.g., salt, $\mathrm{pH}$ ) [77-79], both important pressures in the gut [80-82]. Of the three targets, $d g o R$ is the most likely candidate for the adaptive mutation with which the $d n a Q$ mutator allele could have hitchhiked, since $d g o R$ is mutated in clones from all other mice, but only in the mutator clones from mouse 1. Furthermore, $d g o R$ is the main target of adaptation when a uropathogenic E. coli colonises laboratory mice of a different genetic background [73]. This suggests that mutations in $d g o R$ are strongly adaptive and such adaptation transcends the specific $E$. coli-mouse strain combinations. Interestingly, the mutations targeting $d g o R$ and $f r l R$ (in this and in previous studies) often involve IS insertions, indels, or nonsense mutations. These are likely to lead to loss of function of the negative regulators and, thus, overexpression of the regulated operons. Such adaptive mutations are expected if galactonate and fructoselysine are present in the gut as limiting resources for E. coli. This form of adaptation has been previously shown to occur in the case of galactitol [64].

After divergence from their common ancestor, the mutators acquired potentially adaptive mutations in the $c a d$ operon, $y c b C$, atoC, $p s u K /$ fruA and fruB, and $t d c A / t d c R$. Of these targets, mutations in the last two have previously been observed during adaptation of $E$. coli to the gut of immune-competent or immunocompromised mice [40,41]. Many of the adaptive targets are functionally important for regulating carbon $(d g o R, f r l R$, ato $C$, and, potentially, the cad operon) [77,83-85] or nitrogen metabolism ( $p t s P$ and $t d c A / t d c R$ ) [79,86,87], stress resistance (ptsP, cad operon, $y c b C$, and, potentially, atoC), and peptidoglycan biogenesis $(y c b C)[77,83-$ 85]. Interestingly, $p t s P$, ato $C$, and the $c a d$ operon appear to be involved in regulating both nutritional competence and stress resistance, which suggests that such mutations may modulate a potential trade-off between nutritional competence and stress resistance, which is well described in in vitro experiments and which is also thought to be important in vivo $[81,82,88]$. Together with $d g o R$, the cad operon is the only other mutational target that occurs in all other mice but is specific of mutator clones in mouse 1 .

As the mutator clones have largely independent evolutionary histories (long terminal branches in Fig 2A), the concept of parallel evolution can also be used to identify adaptive targets that were specific to the mutator lineages. However, as the mutation rate is extremely high, parallel mutations can occur just by chance. Thus, we used simulations and conservative filters to identify genes for which the observed number of nonsynonymous single nucleotide 


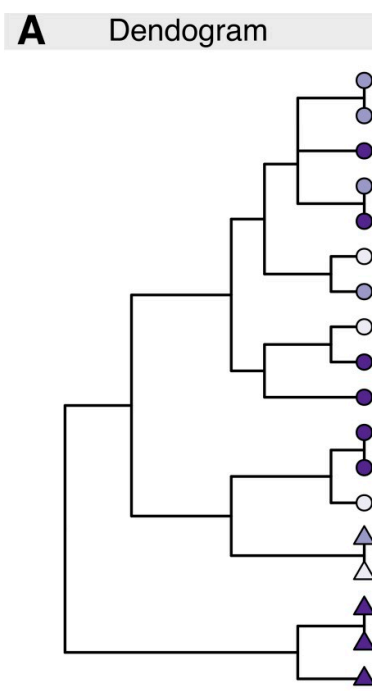

Day post-colonization $\begin{array}{ccc}136 & 148 \quad 190\end{array}$ non-mutators: $\triangle \triangle \Delta$
B Candidate adaptive mutations

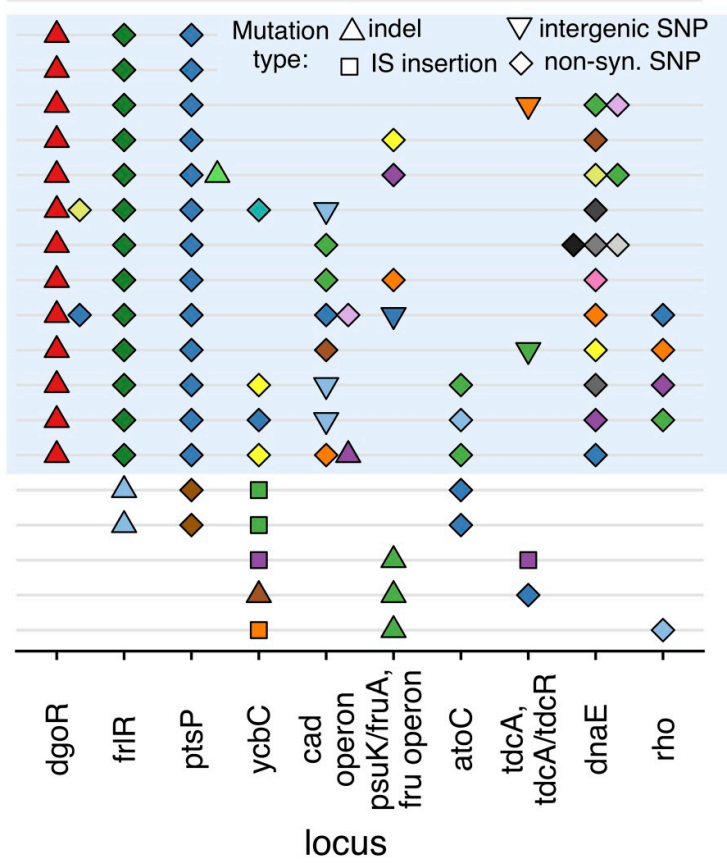

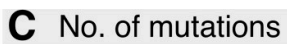

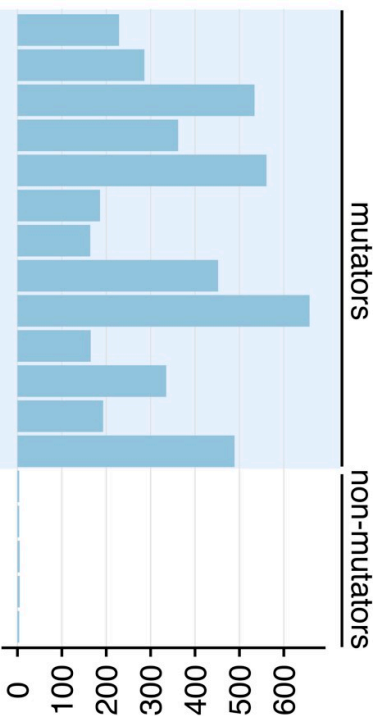

D

Relative frequency and mutation accumulation dynamics for a mutation rate $(U)$ polymorphism under negative frequency-dependent selection

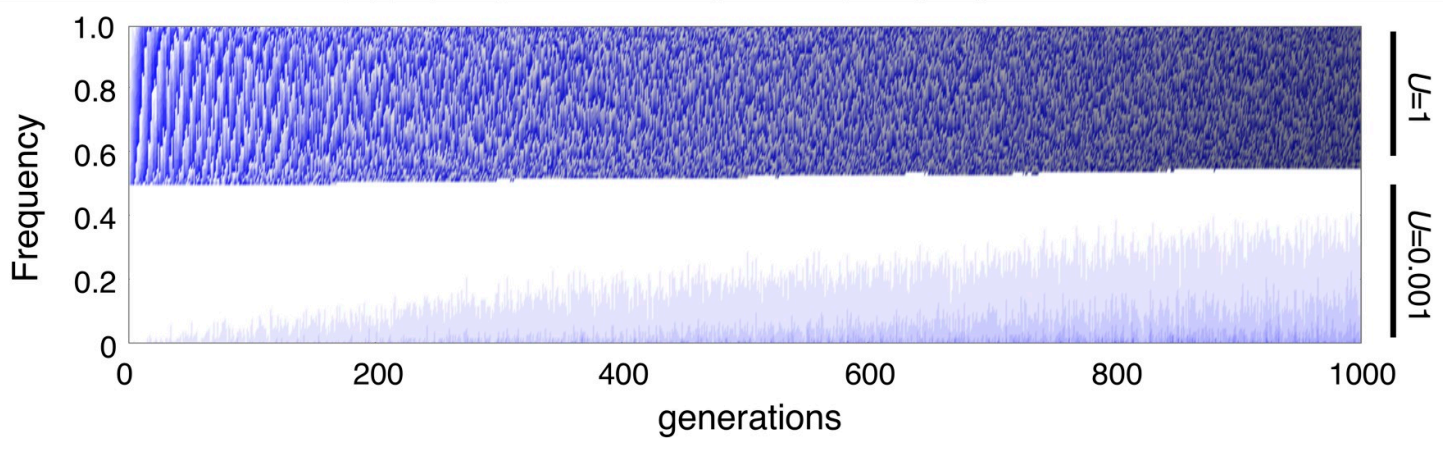

Fig 5. Multiple adaptive mutations accumulate in mutator and nonmutator clones without fixing, highlighting the importance of negative frequency-dependent selection. (A) Clustering of clones depending on which candidate targets of adaptation are mutated. (B) Candidate mutational targets of adaptation that are mutated in each clone (different colours indicate different alleles). All targets that showed parallelism across mice are included, plus $d n a E$ and $r h o$, which were identified from parallelism across mutator clones and for which we have independent evidence of adaptation. Thirty-nine additional targets were identified through parallelism across mutator clones (S6 Table). (C) Number of mutations accumulated per clone. (D) Negative frequency-dependent selection can maintain stable coexistence for 1,000 generations between two clones differing in mutation rate $(U)$ by 1,000 -fold. A population of $10^{6}$ individuals, composed by a 50:50 mixture of two clones with $U=1$ and $U=0.001$ (per genome per generation), was simulated for 1,000 generations, while accumulating deleterious mutations of fixed effect $s_{d}=10^{-5}$. The genotypes of these clones include a locus that is under negative frequency-dependent selection of strength 0.1. Darker tones of grey indicate larger numbers of mutations; shades of blue oscillate every 10 accumulated mutations. The subpopulation with $U=1$ rapidly accumulates many deleterious mutations, reaching about 1,000 mutations by the end of the simulation. In contrast, the majority of the subpopulation with $U=0.001$ remains without any mutations until about 400 generations and has accumulated only up to 3 mutations by generation 1,000. IS, insertion sequence; non-syn., nonsynonymous; SNP, single nucleotide polymorphism.

https://doi.org/10.1371/journal.pbio.3000617.g005

polymorphisms (SNPs) is significantly higher than what would be expected if the observed mutations were randomly distributed (see Methods; [89]). Forty-one genes were identified with this method. Among these, we found $d n a E$ and $c a d C$, which had been identified as adaptive via direct experiments or through parallelism across hosts. Moreover, rho was also identified as a parallel target among mutators and is also mutated in one nonmutator clone, as well 
as during adaptation of adherent-invasive E. coli to the mouse gut [90]. These results suggest that this method is capable of identifying beneficial mutations. Of the identified genes, $>50 \%$ were annotated to the following cluster of orthologous genes (COG) categories: energy production/conversion (5 genes), amino acid transport/metabolism (5 genes), signal transduction (5), and transcription (4). Among the latter two categories, most genes were involved in regulating carbon or amino acid metabolism (S6 Table). This indicates that a large fraction of mutator-specific adaptive mutations are also involved in nitrogen or carbon metabolism, which is in line with the above results.

\section{Partial selective sweeps structure the high polymorphism of a commensal strain evolving in the mouse gut}

Among the tens of adaptive mutations that we identified, none reached fixation. Indeed, even after 190 days of evolution (approximately 3,600 generations), no complete sweep could be observed, neither of a single mutation (hard sweep) nor of multiple, functionally equivalent lineages (soft sweep). This indicates that most beneficial mutations in this ecosystem are only conditionally beneficial. Thus, a more complex form of selection is likely causing the observed polymorphism. This could be mediated by strong clonal interference or spatial or metabolic structuring of niches within the gut and/or caused by forces such as temporally fluctuating or negative frequency-dependent selection (NFDS) (in which the fitness of a genotype increases as its relative abundance decreases). Despite the simplicity of the simulations (Fig 3), these suggest that strong clonal interference is probably not a main mechanism, as simulations with $s_{b}$ $\geq 0.01$ always lead to fixation of one of the competing clones (Figs 3D and S10 and S15), although coexistence is possible when $s_{b} \leq 0.001$ (and $s_{d} \leq 0.0001$ ). However, such low $s_{b}$ values are at odds with the observation of strong mutational parallelism across the four animals and with previous estimates of the fitness effects of beneficial mutations in the mouse gut (which ranged from $2 \%$ to $12 \%$, with an average of $5 \%$ [38-40,64]). A stronger candidate mechanism that could lead to coexistence under a wide range of effects of deleterious mutations is NFDS, which we have previously shown to be operating in the mouse gut $[39,64]$. To query whether NFDS plays a role in the observed dynamics, we tested whether loss of function of $d g o R$ would be under this form of selection ( $d g o R$ is a strong candidate for the driver mutation leading mutators to increase in frequency). When performing in vivo competitions between a $d g o R$ knock-out mutant and the ancestral strain, at two different starting frequencies, we find a signature of NFDS. The $d g o R$ mutant decreases in frequency when it is inoculated at a high frequency but remains at about $10 \%$ when inoculated from a low frequency (S18 Fig). As mutations in $d g o R$ are highly parallel across mice (and even across different $E$. coli-mouse combinations), such mutations must be advantageous when rare. An important role for NFDS is also in complete agreement with three observations: lack of fixation of adaptive mutations (Figs 5 and S17), long-term maintenance of polymorphism for mutation rate (Fig 1), and maintenance of variation at a neutral locus (YFP/CFP; S1 Fig). These are not expected under a regime in which adaptive mutations are unconditionally beneficial but are consistent with a regime in which adaptive mutations are advantageous at low frequency but disadvantageous at high frequency.

To evaluate how NFDS could lead to stable coexistence between two clones with markedly different rates of molecular evolution, we simulated a population with two clones, differing in mutation rate by 1,000 -fold ( $U=1$ and $U=0.001)$, whose genotypes include a locus that is under NFDS of strength 0.1. These clones further acquire recurrent deleterious mutations of effect $s_{d}=10^{-5}$. Under these conditions, the two clones can coexist for at least 1,000 generations at a stable relative frequency of approximately 50\% (Fig 5D; coexistence was unstable in 
the absence of NFDS; Fig 3Ai) and with markedly different patterns of molecular evolution, similar to those observed experimentally (Fig $5 \mathrm{C}$ ). However, we note that a signature of negative frequency-dependent dynamics may also be caused by mechanisms such as temporally fluctuating selection or different metabolic or spatial niches within the gut $[26,91,92]$, beyond the form of NFDS that we simulated (in which fitness of a genotype depends on the relative abundance of another). For example, if there are two independent metabolic niches with different carrying capacities, the dynamics of two genotypes occupying those niches can appear to be under NFDS but may actually be determined by the absolute density of each genotype (as we have previously seen in [64]). A similar argument could be made for spatial niches, as the mammalian gut is inherently spatially structured. The relevance of these mechanisms for maintaining diversity will depend on the rate at which bacterial strains transition between such niches. Thus, further work will be needed in order to understand the relevance of different ecological and evolutionary mechanisms and how the distribution of beneficial and deleterious mutations may change along time, for determining the genetic structure of microbial populations evolving within the gut.

\section{Conclusions}

The emergence and temporal dynamics of mutator populations can provide information on key evolutionary parameters when direct experimental measures may be difficult to obtain. Of key relevance, these can allow the fitness effects of deleterious mutations to be inferred [3135]. Here, we studied the emergence of multiple mutator lineages during colonisation of the mouse gut, including a hypermutator with up to 1,000 -fold increase in mutation rate. Under simplifying assumptions for the distribution of fitness effects of mutations, we found the pattern of molecular evolution to be consistent with effects of deleterious mutations $<10^{-4}$, smaller than in vitro estimates [31-35]. Competitions of MA lines, in vitro and in vivo, also indicate a lower mutational load in the mouse gut than in rich laboratory medium. The mammalian gut is a complex environment that may temporally fluctuate (e.g., variable diet or time of feeding), which could result in temporal variation of the effect of deleterious mutations, with a small time-averaged fitness effect. Indeed, we show in Fig 2D that the availability of particular resources might buffer the fitness effects of deleterious mutations. Moreover, in vitro experiments have shown that the presence of environmental stressors can lead to a reduction in the fitness effect of deleterious mutations [93]. Another mechanism for reducing the effects of deleterious mutations is revealed by the nature of the adaptive mutations. As previously observed, adaptation of $E$. coli to the mouse gut involved mutations linked to carbohydrate metabolism, which appear to be under NFDS, likely created by niche segregation of the different mutants $[39,64]$. This segregation may reduce competition between lineages and therefore buffer the effect of deleterious mutations.

The low effect of deleterious mutations should lead to weak purifying selection, which we detect in the form of a genome-wide $\mathrm{dN} / \mathrm{dS} \approx 1$ (S16 Fig). Analysis of human metagenomics data also shows a $\mathrm{dN} / \mathrm{dS} \approx 1$ for closely related strains with a $\mathrm{dS} \approx 10^{-5}$, similar to the $\mathrm{dS}$ for the mutator clones studied here. However, as is typical for bacteria [94], $\mathrm{dN} / \mathrm{dS}$ declines with increasing $\mathrm{dS}$, reaching $\mathrm{dN} / \mathrm{dS}$ of approximately 0.1 at $\mathrm{dS} \approx 0.01$, indicating strong purifying selection for divergent strains. This might suggest that different forces are affecting the genetic composition of populations on short versus long time scales. Transmission is a key force that is expected to become more important as time passes. Transmission could intensify genetic drift but also increase the strength of purifying selection. For example, deleterious mutations could be lost when transmitted across hosts if their effects are stronger in a new host (relative to the host where these emerged) or if they are hitchhiking with host-specific advantageous 
mutations [5,8]. More generally, understanding the effects of deleterious mutations may be of relevance for a variety of processes both within the gut microbiota (e.g., evolution of antibiotic resistance) and beyond this ecosystem (e.g., cancer evolution). As an example, it has recently been shown that tumour mutational load can be a predictor of survival for some, but not all, types of cancer [95]. Our work suggests that determining the fitness effect of deleterious mutations may be key to understand these differences.

Overall, our work suggests that the evolutionary dynamics of gut commensals may be strongly shaped by beneficial mutations, which increase in frequency but do not fix (partial sweeps), and slightly deleterious mutations that can remain in the population for long periods of time. The combination of these two effects could allow strains of the same species to coexist within the gut microbiota for extended periods with complex temporal dynamics. Novel data on temporal dynamics of genetic polymorphism in species of human gut commensals are starting to confirm this prediction, at least for some species [5-8]. As genetic diversity can impact community composition [96], more time-series data (similar to the data obtained here) are needed for a full understanding of the selective mechanisms that shape microbiota ecoevolutionary dynamics $[97,98]$.

\section{Methods}

\section{Ethics statement}

This research project was ethically reviewed and approved by both the Ethics Committee and the Animal Welfare Body of the Instituto Gulbenkian de Ciência (licence references: A009.2010 and A009.2018) and by the Portuguese National Entity that regulates the use of laboratory animals (Direção Geral de Alimentação e Veterinária [DGAV], licence reference: 009676). All experiments conducted on animals followed the Portuguese (Decreto-Lei ${ }^{\circ}$ 113/ 2013) and European (Directive 2010/63/EU) legislations concerning housing, husbandry, and animal welfare.

\section{Bacterial strains}

Strains used in this manuscript derive from the commensal E. coli K12, substrain MG1655 [99], and are named JB19-YFP, JB18-CFP, RR03-YFP, and RR04-CFP. JB19-YFP and JB18-CFP were previously described in [39]. The four strains carry a streptomycin-resistance mutation RpsL ${ }^{\mathrm{K} 43 \mathrm{R}}$, a deletion of the lac operon ( $\triangle$ lacIZYA), and a fluorescent protein (YFP or CFP) linked to either a chloramphenicol (JB18 and JB19) or ampicillin (RR03 and RR04) resistance cassette that is inserted at the galK locus. Additionally, all strains carry mutations that were previously shown to be adaptive in the mouse gut [38,39]. JB18 and JB19 carry a 1-bp frameshift insertion in gatC. RR03 and RR04 carry the same 1-bp frameshift insertion in $g a t C$, an IS2 insertion, and a 4-bp insertion in the intergenic regions between $y j j P$ and $y j j Q$ and between $y j j Y$ and $y j t D$ (respectively). The mutations $y j j P / y j j Q:: I S 2$ and $y j j Y / y j t D:: 4$ bp were acquired after adapting strain JB19-YFP to the mouse gut for 24 days [39]. P1 transductions [100] were then used to replace the construct with YFP and the chloramphenicol resistance cassette by an ampicillin resistance cassette coupled with either YFP or CFP, thus creating strains RR03-YFP and RR04-CFP.

\section{Mice}

All experiments were carried using 6- to 8-week-old C57BL/6J male mice raised in specific pathogen-free conditions. 


\section{In vivo experimental evolution}

To simulate a perturbation of the mouse gut microbiota and allow its invasion by E. coli, we provided mice with streptomycin-treated water $(5 \mathrm{~g} / \mathrm{L})[38,82]$ for 24 hours before gavage with E. coli and during 1 week post colonisation (Fig 1A). After this period, mice were given normal water until day 190 post colonisation. On the day of gavage, food and water were removed from the cage for 4 hours. Following this period, mice were gavaged with $10^{8} \mathrm{E}$. coli (suspended in $100 \mu \mathrm{l}$ PBS). Four mice were used in this experiment, two of which were gavaged with a mixture of RR03-YFP and JB18-CFP and the other two with a mixture of RR04-CFP and JB19-YFP. Clones RR03/RR04 represented 10\% of the cells in the inoculum, whereas clones JB18/JB19 represented 90\%. These inocula were prepared by growing each clone separately in brain heart infusion (BHI) medium to $\mathrm{OD}_{600 \mathrm{~nm}}=2$ (at $37^{\circ} \mathrm{C}$ on an orbital shaker). After gavage, animals were separated into individual cages, and water and food were returned to them. Colonisation of each mouse was then followed for 190 days. We estimated that 190 days of evolution in the mouse gut correspond to approximately 3,600 generations, assuming 19 generations/day, which was the generation time that we previously measured in streptomycin-treated mice [40]. In this experiment, mice are under streptomycin treatment for 1 week, which reduces microbiota diversity $[28,40]$. Since mice are individually caged and housing conditions impose a high level of sterility, restoration of the microbiota to a high diversity state, which would lead to increases in generation time [101], does not occur.

At each sampling point, faecal pellets were collected, weighed, and dissolved in PBS, with the remaining volume being stored at $-80^{\circ} \mathrm{C}$ in $15 \%$ glycerol. Dilutions were made for plating in LB agar with streptomycin $(100 \mu \mathrm{g} / \mathrm{ml}$; or streptomycin [100 $\mu \mathrm{g} / \mathrm{ml}]+$ ampicillin [100 $\mu \mathrm{g} /$ $\mathrm{ml}] /$ streptomycin $[100 \mu \mathrm{g} / \mathrm{ml}]+$ chloramphenicol $[30 \mu \mathrm{g} / \mathrm{ml}])$ and plates incubated overnight at $37^{\circ} \mathrm{C}$. CFP and YFP colonies were then counted on a fluorescent stereoscope (SteREO Lumar, Carl Zeiss) to estimate total numbers of E. coli and the frequency of YFP and CFP clones (S1 Fig).

\section{Mutator screen and fluctuation tests to estimate mutation rate}

Mutator screen (Fig 1B). Faecal samples isolated from each mouse at day 190 post colonisation were plated as described above. About 90 colonies per mouse were individually picked and inoculated into a different well of a 96-well plate with $200 \mu \mathrm{l}$ of liquid LB. Plates were incubated overnight at $37^{\circ} \mathrm{C}$ on a plate shaker $(800 \mathrm{rpm})$. After the overnight, all clones were frozen at $-80^{\circ} \mathrm{C}$ in $15 \%$ glycerol (generating about 90 clones per mouse). Stored clones were individually defrosted into $200 \mu \mathrm{LB}$ in a 96-well plate and incubated overnight as described above.

Ten microlitres of each culture was removed to quantify the total number of bacteria by FACS, which was approximately $>10^{8}$ per well (only $10 / 430$ samples were between $10^{7}$ and $10^{8}$ ). The remaining volume was plated in $\mathrm{LB}$ agar plates with nalidixic acid $(40 \mu \mathrm{g} / \mathrm{ml})$ to determine the number of resistant mutants that emerged during growth. Plates were incubated as described above.

Fluctuation tests to estimate mutation rate (Figs $1 \mathrm{C}$ and $2 \mathrm{C}$ ). Individual clones, stored at $-80^{\circ} \mathrm{C}$, were defrosted into $\mathrm{PBS}$, plated, and grown overnight at $37^{\circ} \mathrm{C}$. We then picked individual colonies to PBS and used FACS to adjust the inoculum size to 2,000 cells per replicate. Cells were then inoculated into $200 \mu \mathrm{l}$ of liquid LB and grown in a 96-well plate for 20 to 24 hours (incubated at $37^{\circ} \mathrm{C}, 800 \mathrm{rpm}$ in a plate shaker). For some clones, this was done in $1,000 \mu \mathrm{l}$ of liquid LB with growth in 96-deep well plates. At this time point, $10 \mu \mathrm{l}$ of each culture was removed and used to make dilutions to quantify the total number of cells by plating in LB agar plates. The remaining culture volume (and/or a $10^{-1}$ dilution) was then plated in LB plates with rifampicin $(100 \mu \mathrm{g} / \mathrm{ml})$ or nalidixic acid $(40 \mu \mathrm{g} / \mathrm{ml})$ to quantify the number of 
resistant mutants that emerged during growth. All fluctuation tests were repeated in two independent blocks. Across both blocks, 10 replicate growths per clone were carried for all clones in Fig 1C. For the results shown in Figs 2C and S3, 10 replicate growths were carried for the ancestral and the $\mathrm{DnaE}^{\mathrm{T} 771 \mathrm{~S}}$ and $\mathrm{DnaQ}^{\mathrm{L} 145 \mathrm{P}}+\mathrm{DnaE}^{\mathrm{T} 771 \mathrm{~S}}$ clones, whereas 5 replicate growths were carried for each $\mathrm{DnaQ}^{\mathrm{L} 145 \mathrm{P}}$ clones. The total number of colonies and the number of resistant colonies were then used to estimate mutation rate (and 95\% CIs) with FALCOR (using the Ma-Sandri-Sarkar maximum likelihood estimator; [102-106]). This first estimates the number of mutational events $(m)$, which is then divided by the total number of cells $(N t)$, leading to the estimate of mutation rate per cell per generation. Significant differences in mutation rates between clones were identified from nonoverlapping 95\% CIs.

We used fluctuation tests (selecting for antibiotic resistance) to estimate mutation rates, as this allowed measuring the mutation rate for a large number of clones. We scored resistance to rifampicin or nalidixic acid, which arises through SNPs in $r p o B$ or gyrA and gyrB (respectively), thus ignoring mutation rate variation along the genome [70] and neglecting insertions, deletions, and chromosomal rearrangements. The latter mutational types can contribute an important fraction of deleterious mutations, suggesting we may overestimate the deleterious mutation rate (per genome per generation; $U_{d}$ ) of mutators, when we assume that mutator $U_{d}$ increases by the same amount as $U$. We note that $>93 \%$ of the mutations observed in the sequenced mutator clones are SNPs (S16 Fig), indicating that the contribution of other mutational types towards mutation rate is reduced. Moreover, it has also been estimated that singlelocus fluctuation assays can overestimate mutation rates by 6 -fold, relative to estimates obtained from synonymous mutations in adapting populations [107]. Thus, the rates obtained by this assay may be overestimates for both mutator and nonmutator strains. Indeed, comparisons of the mutation rate fold-change between a $\Delta m u t L$ and a wild-type E. coli were broadly consistent between fluctuation tests and MA coupled with whole-genome sequencing [70].

\section{Whole-genome sequencing and analysis}

Sequencing. Nineteen clones were sequenced from mouse 1 (where mutators emerged; 18 CFP and 1 YFP), 3 from mouse 2, 3 from mouse 3 , and 4 from mouse 4 . Bacterial clones were defrosted into liquid LB and grown overnight or into PBS, plated in 2-3 LB agar plates, and incubated for 24 hours at $37^{\circ} \mathrm{C}$. After pelleting the cells, we extracted DNA following the procedure described in [108]. DNA library construction (with the Illumina Nextera XT kit) and sequencing were performed by the IGC genomics facility. Each clone was paired-end sequenced on an Illumina MiSeq Benchtop Sequencer. Standard procedures produced datasets of Illumina paired-end 250-bp read pairs, with mean coverage of 32-fold (19- to 45-fold per clone). The sequences (fastq files) can be found at the NCBI short-read archive (http://www. ncbi.nlm.nih.gov/sra; accession no. PRJNA580181). The ancestral clones had been previously sequenced for [39] (NCBI accession no. SRP063701).

Variant calling. breseq v0.26 [109], with default parameters, was used to map reads and identify mutations, using the E. coli str. K-12 substrain MG1655 genome as reference (NCBI accession: NC_000913.2). Mutations were then determined by comparison with the ancestral strains (JB18, JB19, RR03, RR04, sequenced for [39]). The reads that breseq failed to match to the reference genome were assembled into contigs with SPAdes v.3.13 (using the following parameters $-\mathrm{m} 3$-t 2--careful) [110]. All contigs with coverage $>3$ were then manually annotated via BLAST [111]. All annotated contigs matched the cassettes carrying the antibiotic resistance plus the fluorescent protein (with a single exception, a likely contaminant), suggesting that horizontal gene transfer did not occur during this experiment. 
Phylogeny. We used the COMPARE function of the breseq gdtools suite $[109,112]$ to create a matrix of the genetic differences between each clone. The raw mutational distances between clones were then obtained and used to construct a minimal evolution tree [113] (using the dist.dna and fastme.ols functions from the R package ape, v5.2; [114]), which was plotted with the ggtree function from the R package ggtree (v1.14) [115,116], generating the qualitative trees shown in Figs 2A and S16.

Parallel mutational targets across nonmutator clones (for Fig 5B). These were identified by finding the mutational targets (genes and operons) that independently acquired mutations in more than one nonmutator clone (S17 Fig). As mutations are random and only 3 to 8 mutations accumulated in nonmutator clones, it is very unlikely that we would find the same gene/operon being independently mutated if such mutations did not carry a fitness benefit [117]. Using this strategy, we identified 13 mutational targets that are candidates for adaptation, 12 of which were mutated in clones isolated from different mice and a single one that acquired independent mutations in two clones isolated from the same animal.

Parallel mutations across mutator clones (within mouse 1; for Fig 5B). As mutator clones have long terminal branches (Fig 2A), most of the evolutionary history of each clone is independent of the others. Thus, the mutations accumulating at these terminal branches can be used to identify parallel mutational targets. However, as the mutation rate is up to 1,000 -fold higher than in nonmutator clones, there is the possibility that some mutational targets would accumulate multiple independent mutations just by chance (e.g., longer genes have a higher per-gene probability to acquire a mutation). Hence, we used a statistical approach to identify which genes accumulated more SNPs (at the terminal branches) across independent clones than would be expected by chance. We focused on SNPs, which represent the large majority of the observed mutations (S16 Fig), and on genes, as these are straightforward to define at both the sequence and functional levels. The statistical approach we used is similar to the one in [89]. Briefly, we first simulated 100 datasets in which we randomly distributed the 3,245 independent SNPs (synonymous, nonsynonymous, and intergenic) that we identified in the mutators. This randomisation process was done taking into account the observed number of each specific substitution (i.e., if it is $\mathrm{A} \rightarrow \mathrm{T}$ or $\mathrm{G} \rightarrow \mathrm{C}$, etc.). From these simulations, we kept the nonsynonymous mutations and calculated a $\mathrm{G}$ score for each gene in each simulation. This involved first calculating the expected number of mutations $\left(E_{i}\right)$, as:

$$
E_{i}=N_{t o t}\left(L_{i} / L_{t o t}\right)
$$

where $N_{\text {tot }}$ represents the total number of nonsynonymous SNPs (per simulation), $L_{i}$ is the length of gene $i$, and $L_{\text {tot }}$ is the total size of the coding genome. The $G$ score for gene $i$ can then be calculated as:

$$
G_{i}=2 N_{i} \log _{e}\left(N_{i} / E_{i}\right)
$$

where $N_{i}$ is the number of nonsynonymous SNPs at gene $i$. When $N_{i}=0$, we set $G_{i}=0$. Using the same approach, we calculated $G$ scores for the observed mutations at each of the mutated genes. We could then calculate a $Z$ score for each gene $i$ at which mutations were observed: $Z_{i}=\left(G_{i, o b s}-\overline{G_{i, s i m}}\right) / \operatorname{stdev}\left(G_{i, \text { sim }}\right)$, where $G_{i, \text { obs }}$ and $G_{i, \text { sim }}$ are the $G$ score for the observed mutations and the simulations at gene $i$, respectively (overbar and stdev indicate mean and standard deviation). The $Z_{i}$ scores were then used to calculate Benjamini-Hochberg-corrected $p$-values. Furthermore, we applied two additional conservative criteria: (1) We divided the observed $N_{i}$ and $N_{\text {tot }}$ by 2 before estimating $G_{i, \text { obs }}$. This takes into account that mutation rates can vary along the genome, in general by 2 -fold [118-120]. Dividing $N_{i}$ and $N_{t o t}$ by 2 aims to control for the possibility that significant genes are in regions of elevated mutation rate. (2) 
We included only genes that were mutated in at least 3 independent clones, as this leads to a similar probability of a mutation being parallel as for nonmutator clones, in which two independent mutations are considered (i.e., for nonmutator: $U^{2}=\left[5 \times 10^{-10}\right]^{2}=2.5 \times 10^{-19}$; for mutator: $\left.U^{3}=\left[5 \times 10^{-7}\right]^{3}=1.25 \times 10^{-19}\right)$. This analysis led to the identification of the parallel mutational targets described in S6 Table.

dN/dS estimates. As above, we used the mutations at the terminal branches to estimate $\mathrm{dN} / \mathrm{dS}$ (across the entire genome) for each mutator clone, by taking the ratio of the nonsynonymous to synonymous SNPs. Given that the probability of an SNP to be nonsynonymous is not uniform across the different types of substitutions, we also estimated the expected $\mathrm{dN} / \mathrm{dS}$, given the observed mutational spectra of each clone (using a similar approach to that described in [112]). dN/dS values, shown in S16 Fig, are normalised by the expected value, and $p$-values are obtained with a binomial test.

DNA polymerase III structure (Fig 2B). The DNA polymerase III structure was obtained from rcsb.org (PDB ID: 5M1S) [49,121] and edited with PyMOL to display mutations [122].

\section{Allelic reconstructions and P1 transductions}

The mutations Dna $Q^{\mathrm{L} 145 \mathrm{P}}(\mathrm{CTC} \rightarrow \mathrm{C} C \mathrm{C})$ and $\mathrm{DnaE}^{\mathrm{T} 771 \mathrm{~S}}(\underline{\mathrm{ACG}} \rightarrow \underline{\mathrm{TCG}})$ were constructed by pORTMAGE recombineering [123] in the ancestral (RR04-CFP) background, with the pORTMAGE-3 plasmid (carrying the kanamycin resistance cassette; oligomers are listed in S7 Table). The double mutant was constructed with the same method, by inserting the $\mathrm{DnaQ}^{\mathrm{L} 145 \mathrm{P}}$ mutation in the dnaE background. The presence of these mutations was confirmed by PCR and Sanger sequencing. Subsequently, we grew each clone in LB to lose the pORTMAGE plasmid (this was confirmed by streaking the clones in LB agar plates with or without kanamycin, $100 \mu \mathrm{g} / \mathrm{ml}$ ). P1 transductions were used to construct the following clones: a YFP-expressing DnaQ ${ }^{\mathrm{L} 145 \mathrm{P}}+\mathrm{DnaE}^{\mathrm{T} 771 \mathrm{~S}}$ mutant and a $\Delta d g o R$ mutant. The YFPexpressing DnaQ ${ }^{\mathrm{L} 145 \mathrm{P}}+\mathrm{DnaE}^{\mathrm{T} 771 \mathrm{~S}}$ strain was created by replacing the CFP and ampicillin resistance cassette by a chloramphenicol resistance cassette coupled with YFP. The $\Delta d g o R$ strain was created by replacing the wild-type $d g o R$ in RR04-CFP by the respective knockout from the KEIO collection (strain JW5627; [124]), in which the $d g o R$ sequence is replaced by a kanamycin resistance cassette.

\section{In vivo and in vitro mutation frequency temporal dynamics}

To measure the in vivo mutation frequency (Fig $2 \mathrm{E}$ and $2 \mathrm{~F}$ ), we inoculated different mice with one of three different clones: ancestral (RR04), a DnaQ ${ }^{\mathrm{L} 145 \mathrm{P}}$ single mutant (dnaQ; randomly chosen from the clones with highest mutation rate), and a $\mathrm{DnaQ}^{\mathrm{L} 145 \mathrm{P}}+\mathrm{DnaE}^{\mathrm{T771S}}$ double mutant ( $d n a Q+d n a E$; chosen as for single $d n a Q$ clone). All clones carry the CFP marker.

Mice were provided with streptomycin-treated water $(5 \mathrm{~g} / \mathrm{L})$ for 7 days before gavage with E. coli. Four hours before gavage, food and water were removed from the cages. After gavage, animals were separated into individual cages, and food and normal water (without streptomycin) were returned to them. To prepare the inoculum for these experiments, we defrosted each clone into PBS and plated in LB agar plates. After overnight incubation at $37^{\circ} \mathrm{C}$, colonies were scrapped into PBS and $\mathrm{OD}_{600 \mathrm{~nm}}$ adjusted to 2. One hundred microlitres of this suspension was then used to inoculate each mouse (i.e., $10^{8}$ E. coli). Independent replicate inocula were used to colonise independent mice. This procedure for preparing the inocula is different from the above because we wanted to minimise the strength of selection for compensatory mutations. These could reduce the mutation rate and/or the growth effects of the mutations (growth in liquid media, in which competition is global, is known to lead to more rapid adaptation than in solid media, in which competition is local; [125]). Faecal samples were then obtained at 6 
hours and every 24 hours after gavage for 4 days and suspended in PBS. These were directly plated in LB agar plates with streptomycin and rifampicin to quantify the number of de novo $\mathrm{Rif}^{\mathrm{R}}$ mutants, and dilutions were made to plate in LB agar plates with streptomycin to quantify the total number of $E$. coli (all clones are streptomycin-resistant). These two numbers were then used to estimate the equilibrium mutation frequency, which is proportional to mutation rate (as described in the Results section). Three mice were inoculated with each of the three clones to measure the mutation frequency towards rifampicin (Fig 2E and 2F). This experiment was repeated with two mice per clone, in order to measure the mutation frequency towards nalidixic acid (S6 Fig).

As we had estimated mutation rate in vitro with fluctuation tests, we carried a control experiment to understand if the equilibrium mutation frequency was similar between the in vitro and in vivo conditions. For this, we prepared the inocula of the three different clones as described above but started cultures with $10^{6} \mathrm{E}$. coli in $3 \mathrm{ml}$ liquid LB (in 15-ml tubes). Cultures were incubated for 24 hours at $37^{\circ} \mathrm{C}$ in an orbital shaker. Every 24 hours, cultures were diluted 1,000-fold and allowed to grow until saturation (population size $\approx 3$ to $5 \times 10^{9}$; 10 generations per day). This procedure was repeated for 4 passages. Every day, the cells were plated as described for the in vivo experiment. The results of this experiment showed that the mutation frequency for all mutants was similar between the in vitro and the in vivo conditions (S5 Fig).

We used linear mixed models (with the R package nlme, v3.1 [126]) to analyse the temporal dynamics of mutation frequency and bacterial density, with replicate as a random effect. Both mutation frequency and bacterial density were $\log _{10}$ transformed in order to meet the assumptions made by parametric statistics.

\section{In vitro growth assays in different media}

Bacteria were defrosted into $150 \mu$ l of liquid LB, grown overnight in a 96-well plate, and incubated at $37^{\circ} \mathrm{C}$ in a plate shaker (at $800 \mathrm{rpm}$ ). $\mathrm{OD}_{600 \mathrm{~nm}}$ was then adjusted to 0.01 , cultures were diluted 1:100, and $5 \mu \mathrm{l}$ was spotted in agar plates and incubated at $37^{\circ} \mathrm{C}$ for 48 hours. After incubation, growth of each clone was imaged on a fluorescent stereoscope (SteREO Lumar, Carl Zeiss). The same inocula were tested across all media. The media used were LB and M9 minimal media with $0.4 \%$ glucose, $0.4 \%$ sorbitol, and $0.4 \%$ glucose plus the 20 proteinogenic amino acids (each at $0.05 \mathrm{mM}$ ) (Fig 2D).

\section{MA coupled with in vitro and in vivo competitions}

MA. An MA experiment was started by streaking the $\mathrm{DnaQ}^{\mathrm{L} 145 \mathrm{P}}+\mathrm{DnaE}^{\mathrm{T} 771 \mathrm{~S}}$ clone (expressing CFP) on two independent $\mathrm{LB}$ agar plates. Plates were then incubated at $37^{\circ} \mathrm{C}$ for 24 hours. After incubation, a single colony was picked from each plate and streaked in a new LB agar plate. This procedure was repeated for 4 consecutive passages, generating MA lines 1 and 2. After 4 passages, one colony from each independent line was grown overnight in liquid $\mathrm{LB}$ and stored at $-80^{\circ} \mathrm{C}$. We always selected the last visible colony on the streak, in order to ensure that a random colony was selected $[33,34]$. In each streak, colonies grow from a single cell to approximately $10^{8}$ cells, leading to approximately 25 generations per passage.

In vitro and in vivo competitions. The two MA lines (which express $C F P$ ) and the $\mathrm{DnaQ}^{\mathrm{L} 145 \mathrm{P}}+\mathrm{DnaE}^{\mathrm{T} 771 \mathrm{~S}}$ clone (expressing YFP) were defrosted into PBS, plated in BHI plates, and incubated overnight at $37^{\circ} \mathrm{C}$. After incubation, colonies were scraped into $1 \mathrm{ml} \mathrm{PBS}$, and $100 \mu \mathrm{l}$ of this suspension was added to $5 \mathrm{ml}$ of liquid BHI (in 125-ml erlenmeyers). After 4-hour incubation, $\mathrm{OD}_{600 \mathrm{~nm}}$ was measured and the $\mathrm{DnaQ}^{\mathrm{L} 145 \mathrm{P}}+\mathrm{DnaE}^{\mathrm{T} 771 \mathrm{~S}}-\mathrm{YFP}$ clone was mixed with each of the MA lines, at a concentration of $10^{9}$ cells per ml, with a ratio of 5:1 of 
the MA line to the $\mathrm{DnaQ}{ }^{\mathrm{L} 145 \mathrm{P}}+\mathrm{DnaE}^{\mathrm{T} 771 \mathrm{~S}}$-YFP clone. This was done because preliminary experiments showed that a 1:1 ratio would lead to the MA lines being represented at $<10 \%$ to $20 \%$ frequency in the inoculum.

For in vitro competitions, a $10^{-5}$ dilution of the suspension above was done, and $150 \mu \mathrm{l}$ of this was then added to a 96-well plate and incubated for 24 hours (4 replicates per MA line). The inoculum and the 24-hour time point were then plated in LB agar plates with streptomycin in order to estimate the numbers of CFP and YFP colonies.

For in vivo competitions, $100 \mu \mathrm{l}$ of the above suspension (about $10^{8}$ cells) was gavaged into mice ( 3 mice were gavaged per competition, but for line 1, only two mice became colonised).

Faecal samples were then collected at 6 hours and days 1,2, and 3 post gavage and plated in LB agar plates with streptomycin. As above, mice were provided with streptomycin-treated water $(5 \mathrm{~g} / \mathrm{L})$ for 7 days before gavage with $E$. coli. On the day of gavage, food and water were removed from the cages for the 4 -hour preceding gavage. After gavage, food and normal water (without streptomycin) were returned to all animals. All mice were single-caged for the duration of the experiment.

To determine the fitness decline (i.e., selection coefficient) of each of the MA lines, we first calculated the natural logarithm of the ratio of the evolved (MA line) against the ancestral $\left(\mathrm{DnaQ}^{\mathrm{L} 145 \mathrm{P}}+\mathrm{DnaE}^{\mathrm{T} 771 \mathrm{~S}}-\mathrm{YFP}\right)$ at each time point. The fitness decline is then the slope of this value along time (in generations). In the in vitro experiments, the number of generations was directly estimated from the data by taking the natural logarithm of the ratio between the number of cells of the ancestral at 24 hours relative to that in the inoculum. In in vivo experiments, 19 generations per day were assumed, as has been determined in [40]. As MA line 1 went below detection level in 3 out of 4 replicates, we replaced the 0 counts by 1 , in order to be able to estimate a minimum selection coefficient for these replicates.

We used linear mixed models (with the R package nlme, v3.1 [126]) to analyse the fitness decline (per generation), with MA line as a random effect.

\section{In vivo competitions to test for NFDS on $\Delta d g o R$}

Mice received streptomycin-treated water for 7 days before gavage and were gavaged as described above. Inocula for gavage were prepared by mixing the $\Delta d g o R$ mutant with the ancestral (RR03-YFP) at a ratio of 9:1 or 1:9.

\section{Statistical analysis}

All analyses were performed in R, version 3.5.1 [127], using the statistical methods described in the previous sections.

\section{Simulations of evolving populations}

Simulations were written in Mathematica [128] or C++. The annotated simulation codes are freely available online from Figshare at https://doi.org/10.6084/m9.figshare.10048427.v1.

Simulations of evolving clonal populations that can acquire deleterious mutations of fixed effects were implemented similar to those in [61]. Beginning with a mutation-free population of $10^{5}$ or $10^{6}$ individuals, at each generation mutations were drawn from a Poisson distribution with parameter $U$ (mutation rate). The fitness of each individual was computed as $w_{i}=\left(1-s_{d}\right)^{i}$, where $i$ is the number of mutations carried, and $s_{d}>0$ is the deleterious selection coefficient. The next generation of the population was obtained by sampling $10^{6}$ individuals from the current population according to probabilities weighed by the individuals' absolute fitness. After 1,000 and 2,000 generations, respectively, we recorded the minimum number of mutations accumulated within the population (i.e., number of mutations in the least-loaded class), the 
mean and standard deviation of the number of mutations carried across the whole population, and the mean and standard deviation of fitness within the population.

For the model that includes neutral mutations, we performed the same simulations with two classes of mutations, a deleterious class with mutation rate $U^{*}\left(1-f_{\text {neut }}\right)$ and selection coefficient $s_{d}$ and a neutral class with mutation rate $U^{*} f_{\text {neut }}$ and selection coefficient 0 .

For the model with a continuous distribution of fitness effects (Figs 3B and 3D and S8, S9, S10, S12, S13, and S15), upon mutation, an individual fitness effect per mutation was drawn from an exponential distribution with mean $s_{d}$. When including a proportion of beneficial mutations, these were drawn from an exponential distribution with mean $s_{b}$. As described for the model with neutral mutation, the simulation was performed with two classes of mutations, and the total fitness was computed as the product of the individual fitnesses $1+s_{b, i}$ and $1+s_{d, i}$ of all beneficial and deleterious mutations an individual carried, respectively.

For any parameter combination, we considered that the number of mutations in the simulations fitted those in the experiments if the number of mutations in the simulations was contained within the range of the number of observed mutations across clones (Figs $3 \mathrm{~A}$ and $3 \mathrm{~B}$ and S7, S8, S9, S11, S12, and S13).

For simulations of two subpopulations with different mutation rates (Figs $3 \mathrm{C}$ and $3 \mathrm{D}$ and S10, S14, and S15), a marker locus was implemented that determines the mutation rate within the subpopulation built by its carriers. The number of individuals of subpopulation 1 in the next generation $N_{1}(t+1)$ was sampled from a binomial distribution with parameters $N=10^{6}$ and $p_{1}(t+1)=\frac{W_{1}}{\left(W_{1}+W_{2}\right)}$, where $W_{k}$ is the total fitness of subpopulation $k$ at generation $t$ (i.e., the sum of all current individual fitnesses in this subpopulation). The number of individuals in subpopulation 2 was then determined as $N_{2}(t+1)=N-N_{1}(t+1)$. These simulations were performed both for fixed-effect deleterious mutations (with or without neutral mutations) and for an exponential distribution of fitness effects.

Finally, for simulations with NFDS (Fig 5D), the number of individuals of subpopulation 1 in the next generation $N_{1}(t+1)$ was sampled from a binomial distribution with parameters $N$ $=10^{6}$ and

$$
p_{1}(t+1)=\frac{\left(1+s_{\text {freq }}\left(1-p_{1}(t)\right)\right) W_{1}}{\left(\left(1+s_{\text {freq }}\left(1-p_{1}(t)\right)\right) W_{1}+\left(1+s_{\text {freq }} p_{1}(t)\right) W_{2}\right)},
$$

where $s_{\text {freq }}=0.1$.

\section{Supporting information}

S1 Fig. Temporal dynamics of E. coli densities and frequency of two clones over 190 days (A) and colony size variation at day 190 (B). Mouse 1 is where mutators emerged. Clones used to colonise mice 1 and 2: YFP, gatC::+C (yellow); CFP, gatC::+C, yjjP/yjjQ::IS2, yjjY/yjtD:: +TTAT (blue). Clones used to colonise mice 3 and 4 have the same genotype, but the markers are swapped. Specifically, mice 3 and 4 were colonised with CFP, gatC::+C (blue); YFP, gatC:: +C, yjjP/yjjQ::IS2, yjjY/yjtD::+TTAT (yellow). CFP, cyan fluorescent protein; YFP, yellow fluorescent protein.

S2 Fig. Mutators accumulate mutations in multiple genes that can affect mutation rate. Each row represents a clone, and points with different colours represent different alleles. Genes were identified from Ecocyc (by looking at genes involved in DNA repair and replication) [129] and from [50,130-133].

(TIF) 
S3 Fig. In vitro mutator strengths, estimated from fluctuation tests, for the Anc, DnaQ $Q^{\mathrm{L145P}}$, $\mathrm{DnaE}^{\mathrm{T} 771 \mathrm{~S}}$, DnaQ ${ }^{\mathrm{L145P}}+\mathrm{DnaE}^{\mathrm{T} 771 \mathrm{~S}}$. Data for Anc, DnaQ ${ }^{\mathrm{L} 145 \mathrm{P}}$, and $\mathrm{DnaQ}^{\mathrm{L} 145 \mathrm{P}}+\mathrm{DnaE}^{\mathrm{T} 771 \mathrm{~S}}$ are the same as in Fig 2C. These are repeated here to enable visual comparison. For ease of visualisation, $95 \%$ CIs are only shown for the ancestral and $\mathrm{DnaE}^{\mathrm{T} 771 \mathrm{~S}}$, but none of the $95 \%$ CIs of the $\mathrm{DnaQ}^{\mathrm{L} 145 \mathrm{P}}$ or the DnaQ ${ }^{\mathrm{L} 145 \mathrm{P}}+\mathrm{DnaE}^{\mathrm{T} 771 \mathrm{~S}}$ mutants overlap with either the ancestral or the $\mathrm{DnaE}^{\mathrm{T} 771 \mathrm{~S}}$ mutant (see $\mathrm{S} 3 \mathrm{Table}$ for mutation rates and 95\% CI). Anc, ancestral clone. (TIF)

S4 Fig. In vitro growth capacity of additional clones with mutations in DnaQ ${ }^{\mathrm{L} 145 \mathrm{P}}$, $\mathrm{DnaE}^{\mathrm{T} 771 \mathrm{~S}}$, and $\mathrm{DnaQ}^{\mathrm{L} 145 \mathrm{P}}+\mathrm{DnaE}^{\mathrm{T} 771 \mathrm{~S}}$.

S5 Fig. Mutation frequency has similar dynamics in vitro and in vivo. Top: Temporal dynamics for the frequency of rifampicin-resistant mutants during an in vitro propagation in LB (left) and in vivo colonisation of the mouse gut (right) with the ancestral, Dna $Q^{\mathrm{L} 145 \mathrm{P}}$ mutant, and $D n a Q^{\mathrm{L} 145 \mathrm{P}}+\mathrm{DnaE}^{\mathrm{T} 771 \mathrm{~S}}$ double mutant. Bottom: Box plots showing the pooled data points across all time points (colours represent different clones and shapes represent different replicates). Using a linear mixed model (with replicate as random effect), we find that there is no significant effect of either the interaction between clone and environment (i.e., in vitro or in vivo; $\left.\chi_{2}^{2}=1.96, p=0.38\right)$ or of experiment $\left(\chi_{1}^{2}=0.49, p=0.48\right)$, with the clone being the only significant effect $\left(\chi_{2}^{2}=45.06, p<0.0001\right)$. LB, lysogeny broth.

S6 Fig. (A) In vivo dynamics and summary box plots of the mutation frequency towards nalidixic acid resistance, relative to the average mutation frequency obtained for the ancestral (i.e., in vivo mutator strength). (B) In vivo dynamics and summary box plots of E. coli CFU per gram of faeces. CFU, colony-forming units.

S7 Fig. Simulations of a monoclonal population of $10^{6}$ individuals. Numbers indicate the mean number of mutations, and colour gradient indicates mean fitness after 1,000 (top) and 2,000 generations (bottom; initial fitness is 1). Mutations were either all deleterious with a fixed fitness effect (left) or neutral, with the fraction of neutral mutations $\left(f_{\text {neut }}\right)$ being $50 \%$ or $90 \%$ (middle and right panels; $n=10$ simulations per parameter combination when $f_{\text {neut }}=0$; $n$ $=3$ simulations per parameter combination when $f_{\text {neut }}=0.5$ or $f_{\text {neut }}=0.9$ ). See S4 Table for the mean and standard deviation of fitness and the number of mutations.

(TIF)

S8 Fig. Simulations of a monoclonal population of $10^{6}$ individuals. Numbers indicate the mean number of mutations, and colour gradient indicates mean fitness after 1,000 (top) and 2,000 generations (bottom; initial fitness is 1). All mutations are deleterious, either with a fixed fitness effect $\left(s_{d}\right.$; left; $n=10$ simulations per parameter combination) or with exponentially distributed effects with mean $s_{d}$ (right; $n=3$ simulations per parameter combination).

S9 Fig. Simulations of a monoclonal population of $10^{6}$ individuals. Either mutations were all deleterious $\left(f_{b e n}=0\right)$ or a fraction $\left(f_{b e n}\right)$ of $1 \%(\mathrm{~A})$ or $10 \%(\mathrm{~B})$ was beneficial. The fitness effects of deleterious $\left(s_{d}\right)$ and beneficial $\left(s_{b}\right)$ mutations were drawn from two independent exponential distributions. Numbers indicate the mean number of mutations, and the colour gradient indicates mean fitness after 1,000 (top row) and 2,000 generations (bottom row; fitness starts at 1). Different $s_{b}$ values are represented in different columns $(n=3$ simulations per parameter combination). See S5 Table for the mean and standard deviation of fitness and the 
number of mutations.

(TIF)

S10 Fig. Temporal dynamics of competition between two clones differing in mutation rate by 1,000 -fold $\left(U=1\right.$ and $U=0.001$ per genome per generation; equivalent to the DnaQ ${ }^{\mathrm{L} 145 \mathrm{P}}$ mutant and the ancestral, respectively). All populations had $10^{6}$ individuals, and either mutations were all deleterious (left column) or a fraction $\left(f_{\text {ben }}\right)$ of $1 \%$ (top) or $10 \%$ (bottom row) was beneficial. The fitness effects of deleterious $\left(s_{d}\right)$ and beneficial $\left(s_{b}\right)$ mutations were drawn from two independent exponential distributions.

S11 Fig. Simulations of a monoclonal population of $10^{5}$ individuals. Numbers indicate the mean number of mutations, and colour gradient indicates mean fitness after 1,000 (top) and 2,000 generations (bottom; fitness starts at 1). Mutations were either all deleterious with a fixed fitness effect (left) or neutral, with the fraction of neutral mutations $\left(f_{\text {neut }}\right)$ being $50 \%$ or $90 \%$ (middle and right panels; $n=10$ simulations per parameter combination when $f_{\text {neut }}=0 ; n$ $=3$ simulations per parameter combination when $f_{\text {neut }}=0.5$ or $f_{\text {neut }}=0.9$ ). See S8 Table for the mean and standard deviation of fitness and the number of mutations.

S12 Fig. Simulations of a monoclonal population of $10^{5}$ individuals. Numbers indicate the mean number of mutations, and colour gradient indicates mean fitness after 1,000 (top) and 2,000 generations (bottom; fitness starts at 1). All mutations are deleterious, either with a fixed fitness effect ( $s_{d}$; left; $n=10$ simulations per parameter combination) or with exponentially distributed effects with mean $s_{d}$ (right; $n=3$ simulations per parameter combination). (TIF)

S13 Fig. Simulations of a monoclonal population of $10^{\mathbf{5}}$ individuals. Either mutations were all deleterious $\left(f_{\text {ben }}=0\right)$ or a fraction $\left(f_{\text {ben }}\right)$ of $1 \%(\mathrm{~A})$ or $10 \%(\mathrm{~B})$ was beneficial. The fitness effects of deleterious $\left(s_{d}\right)$ and beneficial $\left(s_{b}\right)$ mutations were drawn from two independent exponential distributions. Numbers indicate the mean number of mutations, and the colour gradient indicates mean fitness after 1,000 (top row) and 2,000 generations (bottom row; fitness starts at 1$)$. Different $s_{b}$ values are represented in different columns $(n=3$ simulations per parameter combination). See S9 Table for the mean and standard deviation of fitness and the number of mutations.

S14 Fig. Temporal dynamics of competition between two clones differing in mutation rate by 1,000 -fold $\left(U=1\right.$ and $U=0.001$ per genome per generation; equivalent to the DnaQ $Q^{\mathrm{L} 145 \mathrm{P}}$ mutant and the ancestral, respectively). All populations had $10^{5}$ individuals, and mutations were either all deleterious with a fixed fitness effect (left) or neutral, with the fraction of neutral mutations $\left(f_{\text {neut }}\right)$ being $50 \%$ or $90 \%$ (middle and right panels; $n=3$ simulations per parameter combination).

S15 Fig. Temporal dynamics of competition between two clones differing in mutation rate by 1,000 -fold $\left(U=1\right.$ and $U=0.001$ per genome per generation; equivalent to the DnaQ ${ }^{\mathrm{L} 145 \mathrm{P}}$ mutant and the ancestral, respectively). All populations had $10^{5}$ individuals, and either mutations were all deleterious (left column) or a fraction $\left(f_{\text {ben }}\right)$ of $1 \%$ (top) or $10 \%$ (bottom row) was beneficial. The fitness effects of deleterious $\left(s_{d}\right)$ and beneficial $\left(s_{b}\right)$ mutations were drawn from two independent exponential distributions.

(TIF) 
S16 Fig. Molecular phenotypes of mutator clones. (A) Phylogenetic tree for the mutator clones sequenced from mouse 1. (B) dN/dS with colours highlighting whether this is significantly different from neutral expectation. (C) Mutational spectra. Mutations accumulated at the tips were chosen for this analysis as a way to avoid counting the same mutation multiple times.

S17 Fig. Mutations observed across nonmutator clones isolated from the four different mice ( 3 to 6 clones per mouse). Circles represent genomes, different coloured circles represent different mice (orange, mouse 1; blue, mouse 2; yellow, mouse 3; green, mouse 4). Grey rectangles highlight mutations that were parallel across different mice (gene labels in black) or for which multiple alleles were found within the same mouse (gene labels in grey). Small rectangles crossing a particular circle indicate mutations, with colours representing different mutation types. Note that for the figures in the main text, we always show 5 nonmutator clones from mouse 1, and there are 6 in this figure. This is because we sequenced one clone at day 190 from the YFP background. As this is not genetically related to the ancestral from which the mutators emerged, we do not show this clone in the main figures. YFP, yellow fluorescent protein.

S18 Fig. In vivo competition between the $\Delta d g o R$ mutant and the ancestral, when starting from high or low frequency ( $n=1$ mouse at each starting frequency). The selective coefficient per generation $(\mathrm{S}(\mathrm{gen}))$ is indicated above each line.

(TIF)

S1 Table. Mutations common to all mutator clones, i.e., present in the mutator common ancestor. Mutation in $d n a Q$ is highlighted in bold, as this is the mutation responsible for the increase in mutation rate.

(XLSX)

S2 Table. Mutations identified by breseq across all clones. (XLSX)

S3 Table. Mutation rates and CIs for Figs 2C and S3. Clones in bold were used to estimate mutation frequency in vivo (both $d n a Q$ and $d n a Q+d n a E$ ) and for the mutation accumulation $(d n a Q+d n a E)$.

(XLSX)

S4 Table. Simulations of a monoclonal population of $10^{6}$ individuals, accumulating neutral and/or deleterious mutations of fixed effect (data for Fig $3 \mathrm{~A}$ and in S7 Fig). (XLSX)

S5 Table. Simulations of a monoclonal population of $10^{6}$ individuals, accumulating deleterious and beneficial mutations with exponentially distributed effects (data for Figs $3 \mathrm{~B}$ and S9).

(XLSX)

S6 Table. Candidate targets of adaptation, identified by parallelism across mutator clones. Genes shown pass the criteria described in the Methods. dnaE, cadC, and rho are shown in bold, as these were identified as adaptive through independent methods. Genes are annotated to their COG group with EggNOG v4.5.1 [134]. For the clusters Transcription and Signal transduction mechanisms, or if function is unknown or no orthologs are found, we provide a 
function summary from Ecocyc [129] (to enable understanding what are the functions that such genes are regulating).

(XLSX)

S7 Table. List of oligomers and primers used to mutagenise and confirm mutations (through Sanger sequencing) in $d n a Q$ and $d n a E$.

(XLSX)

S8 Table. Simulations of a monoclonal population of $10^{5}$ individuals, accumulating neutral and/or deleterious mutations of fixed effect (data for S11 Fig).

(XLSX)

S9 Table. Simulations of a monoclonal population of $10^{5}$ individuals, accumulating deleterious and beneficial mutations with exponentially distributed effects (data for S13 Fig). (XLSX)

\section{Acknowledgments}

We thank Daniela Gülereşi for experimental assistance and Brian Charlesworth for highlighting to us how weak the deleterious effects of mutations may be. We thank Ana Sousa, Roberto Balbontín, Ana-Hermina Ghenu, and Lounès Chikhi for helpful comments on the manuscript. We acknowledge the technical support of the following IGC facilities: Genomics Unit, Rodent facility, Flow Cytometry facility, and Advanced Imaging facility.

\section{Author Contributions}

Conceptualization: Ricardo S. Ramiro, Isabel Gordo.

Data curation: Ricardo S. Ramiro, Claudia Bank, Isabel Gordo.

Formal analysis: Ricardo S. Ramiro, Claudia Bank, Isabel Gordo.

Funding acquisition: Isabel Gordo.

Investigation: Ricardo S. Ramiro, Paulo Durão, Claudia Bank, Isabel Gordo.

Methodology: Ricardo S. Ramiro, Paulo Durão, Isabel Gordo.

Project administration: Isabel Gordo.

Resources: Isabel Gordo.

Software: Ricardo S. Ramiro, Claudia Bank.

Supervision: Isabel Gordo.

Validation: Ricardo S. Ramiro, Isabel Gordo.

Visualization: Ricardo S. Ramiro, Paulo Durão, Claudia Bank.

Writing - original draft: Ricardo S. Ramiro, Isabel Gordo.

Writing - review \& editing: Ricardo S. Ramiro, Paulo Durão, Claudia Bank, Isabel Gordo.

\section{References}

1. Thompson LR, Sanders JG, McDonald D, Amir A, Ladau J, Locey KJ, et al. A communal catalogue reveals Earth's multiscale microbial diversity. Nature. 2017; 551: 457-463. https://doi.org/10.1038/ nature24621 PMID: 29088705 
2. Dethlefsen L, Relman DA. Incomplete recovery and individualized responses of the human distal gut microbiota to repeated antibiotic perturbation. Proc Natl Acad Sci. 2011; 108: 4554-4561. https://doi org/10.1073/pnas.1000087107 PMID: 20847294

3. David LA, Maurice CF, Carmody RN, Gootenberg DB, Button JE, Wolfe BE, et al. Diet rapidly and reproducibly alters the human gut microbiome. Nature. 2014; 505: 559-563. https://doi.org/10.1038/ nature12820 PMID: 24336217

4. Foster KR, Schluter J, Coyte KZ, Rakoff-Nahoum S. The evolution of the host microbiome as an ecosystem on a leash. Nature. 2017; 548: 43-51. https://doi.org/10.1038/nature23292 PMID: 28770836

5. Garud NR, Good BH, Hallatschek O, Pollard KS. Evolutionary dynamics of bacteria in the gut microbiome within and across hosts. PLoS Biol. 2019; 17: e3000102. https://doi.org/10.1371/journal.pbio. 3000102 PMID: 30673701

6. Yassour M, Vatanen T, Siljander H, Hämäläinen A-M, Härkönen T, Ryhänen SJ, et al. Natural history of the infant gut microbiome and impact of antibiotic treatment on bacterial strain diversity and stability. Sci Transl Med. 2016; 8: 343ra81-343ra81. https://doi.org/10.1126/scitranslmed.aad0917 PMID: 27306663

7. Truong DT, Tett A, Pasolli E, Huttenhower C, Segata N. Microbial strain-level population structure and genetic diversity from metagenomes. Genome Res. 2017; 27: 626-638. https://doi.org/10.1101/gr. 216242.116 PMID: 28167665

8. Zhao S, Lieberman TD, Poyet M, Kauffman KM, Gibbons SM, Groussin M, et al. Adaptive Evolution within Gut Microbiomes of Healthy People. Cell Host Microbe. 2019; 25: 656-667.e8. https://doi.org/ 10.1016/j.chom.2019.03.007 PMID: 31028005

9. Leatham MP, Banerjee S, Autieri SM, Mercado-Lubo R, Conway T, Cohen PS. Precolonized Human Commensal Escherichia coli Strains Serve as a Barrier to E. coli O157:H7 Growth in the StreptomycinTreated Mouse Intestine. Infect Immun. 2009; 77: 2876-2886. https://doi.org/10.1128/IAl.00059-09 PMID: 19364832

10. Picard B, Garcia JS, Gouriou S, Duriez P, Brahimi N, Bingen E, et al. The Link between Phylogeny and Virulence in Escherichia coli Extraintestinal Infection. Infect Immun. 1999; 67: 546-553. PMID: 9916057

11. Mazel D, Dychinco B, Webb VA, Davies J. Antibiotic Resistance in the ECOR Collection: Integrons and Identification of a Novel aad Gene. Antimicrob Agents Chemother. 2000; 44: 1568-1574. https:// doi.org/10.1128/aac.44.6.1568-1574.2000 PMID: 10817710

12. Sousa A, Frazão N, Ramiro RS, Gordo I. Evolution of commensal bacteria in the intestinal tract of mice. Curr Opin Microbiol. 2017; 38: 114-121. https://doi.org/10.1016/j.mib.2017.05.007 PMID: 28591676

13. Drake JW. A constant rate of spontaneous mutation in DNA-based microbes. Proc Natl Acad Sci. 1991; 88: 7160-7164. https://doi.org/10.1073/pnas.88.16.7160 PMID: 1831267

14. Lynch M, Ackerman MS, Gout J-F, Long H, Sung W, Thomas WK, et al. Genetic drift, selection and the evolution of the mutation rate. Nat Rev Genet. 2016; 17: 704-714. https://doi.org/10.1038/nrg. 2016.1044 PMID: 27739533

15. LeClerc JE, Li B, Payne WL, Cebula TA. High Mutation Frequencies Among Escherichia coli and Salmonella Pathogens. Science. 1996; 274: 1208-1211. https://doi.org/10.1126/science.274.5290.1208 PMID: 8895473

16. Couce A, Alonso-Rodriguez N, Costas C, Oliver A, Blázquez J. Intrapopulation variability in mutator prevalence among urinary tract infection isolates of Escherichia coli. Clin Microbiol Infect. 2016; 22: 566.e1-566.e7. https://doi.org/10.1016/j.cmi.2016.03.008 PMID: 27021422

17. Oliver A. High Frequency of Hypermutable Pseudomonas aeruginosa in Cystic Fibrosis Lung Infection. Science. 2000; 288: 1251-1253. https://doi.org/10.1126/science.288.5469.1251 PMID: 10818002

18. Matic I. Highly Variable Mutation Rates in Commensal and Pathogenic Escherichia coli. Science. 1997; 277: 1833-1834. https://doi.org/10.1126/science.277.5333.1833 PMID: 9324769

19. Fijalkowska IJ, Schaaper RM. Mutants in the Exo I motif of Escherichia coli dnaQ: defective proofreading and inviability due to error catastrophe. Proc Natl Acad Sci U S A. 1996; 93: 2856-2861. https:// doi.org/10.1073/pnas.93.7.2856 PMID: 8610131

20. Fijalkowska IJ, Schaaper RM, Jonczyk P. DNA replication fidelity in Escherichia coli: a multi-DNA polymerase affair. FEMS Microbiol Rev. 2012; 36: 1105-1121. https://doi.org/10.1111/j.1574-6976.2012. 00338.x PMID: 22404288

21. Tenaillon O, Toupance B, Le Nagard H, Taddei F, Godelle B. Mutators, population size, adaptive landscape and the adaptation of asexual populations of bacteria. Genetics. 1999; 152: 485-493. PMID: 10353893 
22. Travis JMJ, Travis ER. Mutator dynamics in fluctuating environments. Proc R Soc B Biol Sci. 2002; 269: 591-597. https://doi.org/10.1098/rspb.2001.1902 PMID: 11916475

23. Notley-McRobb L, Seeto S, Ferenci T. Enrichment and elimination of mutY mutators in Escherichia coli populations. Genetics. 2002; 162: 1055-1062. PMID: 12454055

24. Sniegowski PD, Gerrish PJ, Lenski RE. Evolution of high mutation rates in experimental populations of E. coli. Nature. 1997; 387: 703-705. https://doi.org/10.1038/42701 PMID: 9192894

25. Swings T, Van den Bergh B, Wuyts S, Oeyen E, Voordeckers K, Verstrepen KJ, et al. Adaptive tuning of mutation rates allows fast response to lethal stress in Escherichia coli. eLife. 2017; 6: e22939. https://doi.org/10.7554/eLife.22939 PMID: 28460660

26. Thaiss CA, Zeevi D, Levy M, Zilberman-Schapira G, Suez J, Tengeler AC, et al. Transkingdom Control of Microbiota Diurnal Oscillations Promotes Metabolic Homeostasis. Cell. 2014; 159: 514-529. https:// doi.org/10.1016/j.cell.2014.09.048 PMID: 25417104

27. Yatsunenko T, Rey FE, Manary MJ, Trehan I, Dominguez-Bello MG, Contreras M, et al. Human gut microbiome viewed across age and geography. Nature. 2012; 486: 222-227. https://doi.org/10.1038/ nature11053 PMID: 22699611

28. Thompson JA, Oliveira RA, Djukovic A, Ubeda C, Xavier KB. Manipulation of the Quorum Sensing Signal Al-2 Affects the Antibiotic-Treated Gut Microbiota. Cell Rep. 2015; 10: 1861-1871. https://doi.org/ 10.1016/j.celrep.2015.02.049 PMID: 25801025

29. Gordo I, Campos PRA. Evolution of clonal populations approaching a fitness peak. Biol Lett. 2013; 9: 20120239-20120239. https://doi.org/10.1098/rsbl.2012.0239 PMID: 22764110

30. Giraud A, Matic I, Tenaillon O, Clara A, Radman M, Fons M, et al. Costs and benefits of high mutation rates: adaptive evolution of bacteria in the mouse gut. Science. 2001; 291: 2606-2608. https://doi.org/ 10.1126/science. 1056421 PMID: 11283373

31. Kibota TT, Lynch M. Estimate of the genomic mutation rate deleterious to overall fitness in E. coli. Nature. 1996; 381: 694-696. https://doi.org/10.1038/381694a0 PMID: 8649513

32. Trindade S, Perfeito L, Gordo I. Rate and effects of spontaneous mutations that affect fitness in mutator Escherichia coli. Philos Trans R Soc B-Biol Sci. 2010; 365: 1177-1186. https://doi.org/10.1098/ rstb.2009.0287 PMID: 20308092

33. Lind PA, Andersson DI. Whole-genome mutational biases in bacteria. Proc Natl Acad Sci. 2008; 105 : 17878-17883. https://doi.org/10.1073/pnas.0804445105 PMID: 19001264

34. Heilbron K, Toll-Riera M, Kojadinovic M, MacLean RC. Fitness is strongly influenced by rare mutations of large effect in a microbial mutation accumulation experiment. Genetics. 2014; 197: 981-990. https:// doi.org/10.1534/genetics.114.163147 PMID: 24814466

35. Robert L, Ollion J, Robert J, Song X, Matic I, Elez M. Mutation dynamics and fitness effects followed in single cells. Science. 2018; 359: 1283-1286. https://doi.org/10.1126/science.aan0797 PMID: 29590079

36. Ohta T. The Nearly Neutral Theory of Molecular Evolution. Annu Rev Ecol Syst. 1992; 23: 263-286. https://doi.org/10.1146/annurev.es.23.110192.001403

37. Gillespie JH. Genetic Drift in an Infinite Population: The Pseudohitchhiking Model. Genetics. 2000; 155: 909-919. PMID: 10835409

38. Barroso-Batista J, Sousa A, Lourenço M, Bergman M-L, Sobral D, Demengeot J, et al. The First Steps of Adaptation of Escherichia coli to the Gut Are Dominated by Soft Sweeps. PLoS Genet. 2014; 10: e1004182. https://doi.org/10.1371/journal.pgen.1004182 PMID: 24603313

39. Lourenço M, Ramiro RS, Güleresi D, Barroso-Batista J, Xavier KB, Gordo I, et al. A Mutational Hotspot and Strong Selection Contribute to the Order of Mutations Selected for during Escherichia coli Adaptation to the Gut. PLoS Genet. 2016; 12: e1006420. https://doi.org/10.1371/journal.pgen.1006420 PMID: 27812114

40. Barroso-Batista J, Demengeot J, Gordo I. Adaptive immunity increases the pace and predictability of evolutionary change in commensal gut bacteria. Nat Commun. 2015; 6: 8945. https://doi.org/10.1038/ ncomms9945 PMID: 26615893

41. Frazão N, Sousa A, Lässig M, Gordo I. Horizontal gene transfer overrides mutation in Escherichia coli colonizing the mammalian gut. Proc Natl Acad Sci. 2019; 116: 17906-17915. https://doi.org/10.1073/ pnas. 1906958116 PMID: 31431529

42. Sprouffske K, Aguilar-Rodríguez J, Sniegowski P, Wagner A. High mutation rates limit evolutionary adaptation in Escherichia coli. PLoS Genet. 2018; 14: e1007324. https://doi.org/10.1371/journal.pgen. 1007324 PMID: 29702649

43. Wielgoss S, Barrick JE, Tenaillon O, Wiser MJ, Dittmar WJ, Cruveiller S, et al. Mutation rate dynamics in a bacterial population reflect tension between adaptation and genetic load. Proc Natl Acad Sci. 2013; 110: 222-227. https://doi.org/10.1073/pnas.1219574110 PMID: 23248287 
44. Baym M, Lieberman TD, Kelsic ED, Chait R, Gross R, Yelin I, et al. Spatiotemporal microbial evolution on antibiotic landscapes. Science. 2016; 353: 1147-1151. https://doi.org/10.1126/science.aag0822 PMID: 27609891

45. Gentile Christopher F., Yu Szi-Chieh, Serrano Sebastian Akle, Gerrish Philip J., Sniegowski Paul D. Competition between high- and higher-mutating strains of Escherichia coli. Biol Lett. 2011; 7: 422424. https://doi.org/10.1098/rsbl.2010.1036 PMID: 21227974

46. Echols H, Lu C, Burgers PM. Mutator strains of Escherichia coli, mutD and dnaQ, with defective exonucleolytic editing by DNA polymerase III holoenzyme. Proc Natl Acad Sci U S A. 1983; 80: 2189-2192. https://doi.org/10.1073/pnas.80.8.2189 PMID: 6340117

47. Maki H, Horiuchi T, Kornberg A. The polymerase subunit of DNA polymerase III of Escherichia coli. I. Amplification of the dnaE gene product and polymerase activity of the alpha subunit. J Biol Chem. 1985; 260: 12982-12986. PMID: 2932432

48. Studwell-Vaughan PS, O'Donnell M. DNA polymerase III accessory proteins. V. Theta encoded by holE. J Biol Chem. 1993; 268: 11785-11791. PMID: 8505306

49. Fernandez-Leiro R, Conrad J, Yang J-C, Freund SMV, Scheres SHW, Lamers MH. Self-correcting mismatches during high-fidelity DNA replication. Nat Struct Mol Biol. 2017; 24: 140-143. https://doi. org/10.1038/nsmb.3348 PMID: 28067916

50. Taft-Benz SA, Schaaper RM. The $\theta$ Subunit of Escherichia coli DNA Polymerase III: a Role in Stabilizing the $\varepsilon$ Proofreading Subunit. J Bacteriol. 2004; 186: 2774-2780. https://doi.org/10.1128/JB.186.9. 2774-2780.2004 PMID: 15090519

51. Schaaper RM, Cornacchio R. An Escherichia coli dnaE mutation with suppressor activity toward mutator mutD5. J Bacteriol. 1992; 174: 1974-1982. https://doi.org/10.1128/jb.174.6.1974-1982.1992 PMID: 1548237

52. Maharjan RP, Ferenci T. A shifting mutational landscape in 6 nutritional states: Stress-induced mutagenesis as a series of distinct stress input-mutation output relationships. PLoS Biol. 2017; 15: e2001477. https://doi.org/10.1371/journal.pbio.2001477 PMID: 28594817

53. Haldane JBS. The Effect of Variation of Fitness. Am Nat. 1937; 71: 337-349. https://doi.org/10.1086/ 280722

54. Goldstein BP. Resistance to rifampicin: a review. J Antibiot (Tokyo). 2014; 67: 625-630. https://doi org/10.1038/ja.2014.107 PMID: 25118103

55. Redgrave LS, Sutton SB, Webber MA, Piddock LJV. Fluoroquinolone resistance: mechanisms, impact on bacteria, and role in evolutionary success. Trends Microbiol. 2014; 22: 438-445. https://doi.org/10. 1016/j.tim.2014.04.007 PMID: 24842194

56. Karami-Zarandi M, Douraghi M, Vaziri B, Adibhesami H, Rahbar M, Yaseri M. Variable spontaneous mutation rate in clinical strains of multidrug-resistant Acinetobacter baumannii and differentially expressed proteins in a hypermutator strain. Mutat Res Mol Mech Mutagen. 2017;800-802: 37-45. https://doi.org/10.1016/j.mrfmmm.2017.06.002 PMID: 28646746

57. Peck JR. A Ruby in the Rubbish: Beneficial Mutations, Deleterious Mutations and the Evolution of Sex. Genetics. 1994; 137: 597-606. PMID: 8070669

58. Bachtrog D, Gordo I. Adaptive evolution of asexual populations under Muller's ratchet. Evolution. 2004; 58: 1403-1413. https://doi.org/10.1111/j.0014-3820.2004.tb01722.x PMID: 15341144

59. Muller HJ. The relation of recombination to mutational advance. Mutat Res. 1964; 1: 2-9.

60. Felsenstein J. The evolutionary advantage of recombination. Genetics. 1974; 78: 737-756. PMID: 4448362

61. Gordo I, Charlesworth B. The degeneration of asexual haploid populations and the speed of Muller's ratchet. Genetics. 2000; 154: 1379-1387. PMID: 10757777

62. Lynch M, Gabriel W. Mutation Load and the Survival of Small Populations. Evolution. 1990; 44: 17251737. https://doi.org/10.1111/j.1558-5646.1990.tb05244.x PMID: 28567811

63. Perfeito L, Fernandes L, Mota C, Gordo I. Adaptive mutations in bacteria: High rate and small effects. Science. 2007; 317: 813-815. https://doi.org/10.1126/science.1142284 PMID: 17690297

64. Sousa A, Ramiro RS, Barroso-Batista J, Güleresi D, Lourenço M, Gordo I. Recurrent Reverse Evolution Maintains Polymorphism after Strong Bottlenecks in Commensal Gut Bacteria. Mol Biol Evol. 2017; 34: 2879-2892. https://doi.org/10.1093/molbev/msx221 PMID: 28961745

65. Eyre-Walker A, Keightley PD. The distribution of fitness effects of new mutations. Nat Rev Genet. 2007; 8: 610-618. https://doi.org/10.1038/nrg2146 PMID: 17637733

66. Loewe L, Charlesworth B, Bartolomé C, Nöel V. Estimating Selection on Nonsynonymous Mutations. Genetics. 2006; 172: 1079-1092. https://doi.org/10.1534/genetics.105.047217 PMID: 16299397 
67. Tenaillon O, Skurnik D, Picard B, Denamur E. The population genetics of commensal Escherichia coli. Nat Rev Microbiol. 2010; 8: 207-217. https://doi.org/10.1038/nrmicro2298 PMID: 20157339

68. Massot M, Couffignal C, Clermont O, D'Humières C, Chatel J, Plault N, et al. Day-to-Day Dynamics of Commensal Escherichia coli in Zimbabwean Cows Evidence Temporal Fluctuations within a HostSpecific Population Structure. Appl Env Microbiol. 2017; 83: e00659-17. https://doi.org/10.1128/AEM. 00659-17 PMID: 28411228

69. Gordon DM, O'Brien CL, Pavli P. Escherichia coli diversity in the lower intestinal tract of humans. Environ Microbiol Rep. 2015; 7: 642-648. https://doi.org/10.1111/1758-2229.12300 PMID: 26034010

70. Lee H, Popodi E, Tang H, Foster PL. Rate and molecular spectrum of spontaneous mutations in the bacterium Escherichia coli as determined by whole-genome sequencing. Proc Natl Acad Sci. 2012; 109: E2774-E2783. https://doi.org/10.1073/pnas.1210309109 PMID: 22991466

71. Chao L, Cox EC. Competition Between High and Low Mutating Strains of Escherichia coli. Evolution. 1983; 37: 125-134. https://doi.org/10.1111/j.1558-5646.1983.tb05521.x PMID: 28568016

72. Taddei F, Radman M, Maynard-Smith J, Toupance B, Gouyon PH, Godelle B. Role of mutator alleles in adaptive evolution. Nature. 1997; 387: 700-702. https://doi.org/10.1038/42696 PMID: 9192893

73. Lescat M, Launay A, Ghalayini M, Magnan M, Glodt J, Pintard C, et al. Using long-term experimental evolution to uncover the patterns and determinants of molecular evolution of an Escherichia coli natural isolate in the streptomycin-treated mouse gut. Mol Ecol. 2017; 26: 1802-1817. https://doi.org/10. 1111/mec.13851 PMID: 27661780

74. Nielsen R. Molecular Signatures of Natural Selection. Annu Rev Genet. 2005; 39: 197-218. https:// doi.org/10.1146/annurev.genet.39.073003.112420 PMID: 16285858

75. Wiame E, Delpierre G, Collard F, Van Schaftingen E. Identification of a pathway for the utilization of the Amadori product fructoselysine in Escherichia coli. J Biol Chem. 2002; 277: 42523-42529. https:// doi.org/10.1074/jbc.M200863200 PMID: 12147680

76. Cooper RA. The utilisation of D-galactonate and D-2-oxo-3-deoxygalactonate by Escherichia coli K12. Biochemical and genetical studies. Arch Microbiol. 1978; 118: 199-206. https://doi.org/10.1007/ bf00415730 PMID: 211976

77. Lee J, Park Y-H, Kim Y-R, Seok Y-J, Lee C-R. Dephosphorylated NPr is involved in an envelope stress response of Escherichia coli. Microbiology. 2015; 161: 1113-1123. https://doi.org/10.1099/mic.0. 000056 PMID: 25701731

78. Reizer J, Reizer A, Merrick MJ, Plunkett G, Rose DJ, Saier MH. Novel phosphotransferase-encoding genes revealed by analysis of the Escherichia coli genome: a chimeric gene encoding an Enzyme I homologue that possesses a putative sensory transduction domain. Gene. 1996; 181: 103-108. https://doi.org/10.1016/s0378-1119(96)00481-7 PMID: 8973315

79. Pflüger-Grau K, Görke B. Regulatory roles of the bacterial nitrogen-related phosphotransferase system. Trends Microbiol. 2010; 18: 205-214. https://doi.org/10.1016/j.tim.2010.02.003 PMID: 20202847

80. Winter SE, Winter MG, Xavier MN, Thiennimitr P, Poon V, Keestra AM, et al. Host-derived nitrate boosts growth of E. coli in the inflamed gut. Science. 2013; 339: 708-711. https://doi.org/10.1126/ science.1232467 PMID: 23393266

81. De Paepe M, Gaboriau-Routhiau V, Rainteau D, Rakotobe S, Taddei F, Cerf-Bensussan N. Trade-off between bile resistance and nutritional competence drives Escherichia coli diversification in the mouse gut. PLoS Genet. 2011; 7: e1002107. https://doi.org/10.1371/journal.pgen.1002107 PMID: 21698140

82. Leatham-Jensen MP, Frimodt-Møller J, Adediran J, Mokszycki ME, Banner ME, Caughron JE, et al. The Streptomycin-Treated Mouse Intestine Selects Escherichia coli envZ Missense Mutants That Interact with Dense and Diverse Intestinal Microbiota. Infect Immun. 2012; 80: 1716-1727. https://doi. org/10.1128/IAI.06193-11 PMID: 22392928

83. Lioliou EE, Kyriakidis DA. The role of bacterial antizyme: From an inhibitory protein to AtoC transcriptional regulator. Microb Cell Factories. 2004; 3: 8. https://doi.org/10.1186/1475-2859-3-8 PMID: 15200682

84. Paradis-Bleau C, Kritikos G, Orlova K, Typas A, Bernhardt TG. A Genome-Wide Screen for Bacterial Envelope Biogenesis Mutants Identifies a Novel Factor Involved in Cell Wall Precursor Metabolism. PLoS Genet. 2014; 10: e1004056. https://doi.org/10.1371/journal.pgen.1004056 PMID: 24391520

85. Kanjee U, Houry WA. Mechanisms of Acid Resistance in Escherichia coli. Annu Rev Microbiol. 2013; 67: 65-81. https://doi.org/10.1146/annurev-micro-092412-155708 PMID: 23701194

86. Reitzer L. Catabolism of Amino Acids and Related Compounds. EcoSal Plus. 2005;1. https://doi.org/ 10.1128/ecosalplus.3.4.7 PMID: 26443507

87. Hagewood BT, Ganduri YL, Datta P. Functional analysis of the tdcABC promoter of Escherichia coli: roles of TdcA and TdcR. J Bacteriol. 1994; 176: 6214-6220. https://doi.org/10.1128/jb.176.20.62146220.1994 PMID: 7928991 
88. Ferenci T. Maintaining a healthy SPANC balance through regulatory and mutational adaptation $57(1)$, 1-8. Mol Microbiol. 2005; 57: 1-8. https://doi.org/10.1111/j.1365-2958.2005.04649.x PMID: 15948944

89. Tenaillon O, Barrick JE, Ribeck N, Deatherage DE, Blanchard JL, Dasgupta A, et al. Tempo and mode of genome evolution in a 50,000-generation experiment. Nature. 2016; 536: 165-170. https://doi.org/ 10.1038/nature18959

90. Elhenawy W, Tsai CN, Coombes BK. Host-Specific Adaptive Diversification of Crohn's Disease-Associated Adherent-Invasive Escherichia coli. Cell Host Microbe. 2019 [cited 2019 Feb 4]. https://doi.org/ 10.1016/j.chom.2018.12.010 PMID: 30683582

91. Meador JP, Caldwell ME, Cohen PS, Conway T. Escherichia coli Pathotypes Occupy Distinct Niches in the Mouse Intestine. Infect Immun. 2014; 82: 1931-1938. https://doi.org/10.1128/IAI.01435-13 PMID: 2456662

92. Donaldson GP, Lee SM, Mazmanian SK. Gut biogeography of the bacterial microbiota. Nat Rev Microbiol. 2016; 14: 20-32. https://doi.org/10.1038/nrmicro3552 PMID: 26499895

93. Kishony R, Leibler S. Environmental stresses can alleviate the average deleterious effect of mutations. J Biol. 2003; 2: 14. https://doi.org/10.1186/1475-4924-2-14 PMID: 12775217

94. Rocha EPC, Smith JM, Hurst LD, Holden MTG, Cooper JE, Smith NH, et al. Comparisons of dN/dS are time dependent for closely related bacterial genomes. J Theor Biol. 2006; 239: 226-235. https:// doi.org/10.1016/j.jtbi.2005.08.037 PMID: 16239014

95. Samstein RM, Lee C-H, Shoushtari AN, Hellmann MD, Shen R, Janjigian YY, et al. Tumor mutational load predicts survival after immunotherapy across multiple cancer types. Nat Genet. 2019; 51: 202. https://doi.org/10.1038/s41588-018-0312-8 PMID: 30643254

96. Roches SD, Post DM, Turley NE, Bailey JK, Hendry AP, Kinnison MT, et al. The ecological importance of intraspecific variation. Nat Ecol Evol. 2018; 2: 57. https://doi.org/10.1038/s41559-017-0402-5 PMID: 29203921

97. Ferreiro A, Crook N, Gasparrini AJ, Dantas G. Multiscale Evolutionary Dynamics of Host-Associated Microbiomes. Cell. 2018; 172: 1216-1227. https://doi.org/10.1016/j.cell.2018.02.015 PMID: 29522743

98. Waldor MK, Tyson G, Borenstein E, Ochman H, Moeller A, Finlay BB, et al. Where Next for Microbiome Research? PLoS Biol. 2015; 13: e1002050. https://doi.org/10.1371/journal.pbio.1002050 PMID: 25602283

99. Blattner FR. The Complete Genome Sequence of Escherichia coli K-12. Science. 1997; 277: 14531462. https://doi.org/10.1126/science.277.5331.1453 PMID: 9278503

100. Lennox ES. Transduction of linked genetic characters of the host by bacteriophage P1. Virology. 1955; 1: 190-206. https://doi.org/10.1016/0042-6822(55)90016-7 PMID: 13267987

101. Rang CU, Licht TR, Midtvedt T, Conway PL, Chao L, Krogfelt KA, et al. Estimation of growth rates of Escherichia coli BJ4 in streptomycin-treated and previously germfree mice by in situ rRNA hybridization. Clin Diagn Lab Immunol. 1999; 6: 434-436. PMID: 10225851

102. Hall BM, Ma C-X, Liang P, Singh KK. Fluctuation AnaLysis CalculatOR: a web tool for the determination of mutation rate using Luria-Delbrück fluctuation analysis. Bioinformatics. 2009; 25: 1564-1565. https://doi.org/10.1093/bioinformatics/btp253 PMID: 19369502

103. Stewart FM. Fluctuation tests: how reliable are the estimates of mutation rates? Genetics. 1994; 137 1139-1146. PMID: 7982567

104. Sarkar S, Ma WT, Sandri G v. H. On fluctuation analysis: a new, simple and efficient method for computing the expected number of mutants. Genetica. 1992; 85: 173-179. https://doi.org/10.1007/ bf00120324 PMID: 1624139

105. Ma WT, Sandri GV, Sarkar S. Analysis of the Luria-Delbrück Distribution Using Discrete Convolution Powers. J Appl Probab. 1992; 29: 255-267. https://doi.org/10.2307/3214564

106. Rosche WA, Foster PL. Determining mutation rates in bacterial populations. Methods San Diego Calif. 2000; 20: 4-17. https://doi.org/10.1006/meth.1999.0901 PMID: 10610800

107. Drake JW. Contrasting Mutation Rates from Specific-Locus and Long-Term Mutation-Accumulation Procedures. G3. 2012; 2: 483-485. https://doi.org/10.1534/g3.111.001842 PMID: 22540039

108. Wilson K. Preparation of Genomic DNA from Bacteria. Current Protocols in Molecular Biology. 2001; 56(1):2.4.1-2.4.5. Available from: https://doi.org/10.1002/0471142727.mb0204s56

109. Deatherage DE, Barrick JE. Identification of mutations in laboratory-evolved microbes from next-generation sequencing data using breseq. Methods Mol Biol Clifton NJ. 2014; 1151: 165-188. https://doi. org/10.1007/978-1-4939-0554-6_12 PMID: 24838886

110. Bankevich A, Nurk S, Antipov D, Gurevich AA, Dvorkin M, Kulikov AS, et al. SPAdes: A New Genome Assembly Algorithm and Its Applications to Single-Cell Sequencing. J Comput Biol. 2012; 19: 455477. https://doi.org/10.1089/cmb.2012.0021 PMID: 22506599 
111. Altschul SF, Gish W, Miller W, Myers EW, Lipman DJ. Basic local alignment search tool. J Mol Biol. 1990; 215: 403-410. https://doi.org/10.1016/S0022-2836(05)80360-2 PMID: 2231712

112. Barrick J. Tutorial: Clonal Samples (Consensus Mode)—breseq 0.30.0 documentation. [cited 2019 Feb 28]. Available from: http://barricklab.org/twiki/pub/Lab/ToolsBacterialGenomeResequencing/ documentation/tutorial_clones.html\#example-2-analyze-rates-and-nature-of-genome-evolution

113. Desper R, Gascuel O. Fast and Accurate Phylogeny Reconstruction Algorithms Based on the Minimum-Evolution Principle. J Comput Biol. 2002; 9: 687-705. https://doi.org/10.1089/ 106652702761034136 PMID: 12487758

114. Paradis E, Schliep K. ape 5.0: an environment for modern phylogenetics and evolutionary analyses in R. Bioinformatics. 2019; 35: 526-528. https://doi.org/10.1093/bioinformatics/bty633 PMID: 30016406

115. Yu G, Lam TT-Y, Zhu H, Guan Y. Two Methods for Mapping and Visualizing Associated Data on Phylogeny Using Ggtree. Mol Biol Evol. 2018; 35: 3041-3043. https://doi.org/10.1093/molbev/msy194 PMID: 30351396

116. Yu G, Smith DK, Zhu H, Guan Y, Lam TT-Y. ggtree: an r package for visualization and annotation of phylogenetic trees with their covariates and other associated data. Methods Ecol Evol. 2017; 8: 2836. https://doi.org/10.1111/2041-210X.12628

117. Barrick JE, Lenski RE. Genome dynamics during experimental evolution. Nat Rev Genet. 2013; 14 : 827-839. https://doi.org/10.1038/nrg3564 PMID: 24166031

118. Dillon MM, Sung W, Lynch M, Cooper VS. Periodic Variation of Mutation Rates in Bacterial Genomes Associated with Replication Timing. mBio. 2018; 9: e01371-18. https://doi.org/10.1128/mBio.0137118 PMID: 30131359

119. Foster PL, Hanson AJ, Lee H, Popodi EM, Tang H. On the Mutational Topology of the Bacterial Genome. G3. 2013; 3: 399-407. https://doi.org/10.1534/g3.112.005355 PMID: 23450823

120. Niccum BA, Lee H, Mohammedlsmail W, Tang H, Foster PL. The Symmetrical Wave Pattern of BasePair Substitution Rates across the Escherichia coli Chromosome Has Multiple Causes. mBio. 2019; 10: e01226-19. https://doi.org/10.1128/mBio.01226-19 PMID: 31266871

121. Berman HM, Westbrook J, Feng Z, Gilliland G, Bhat TN, Weissig H, et al. The Protein Data Bank. Nucleic Acids Res. 2000; 28: 235-242. https://doi.org/10.1093/nar/28.1.235 PMID: 10592235

122. Schrodinger. The PyMOL Molecular Graphics System. Version 1.8 [software]. Schrodinger; 2015. Available from: https://pymol.org/

123. Nyerges Á, Csörgő B, Nagy I, Bálint B, Bihari $P$, Lázár $V$, et al. A highly precise and portable genome engineering method allows comparison of mutational effects across bacterial species. Proc Natl Acad Sci. 2016; 113: 2502-2507. https://doi.org/10.1073/pnas.1520040113 PMID: 26884157

124. Baba T, Ara T, Hasegawa M, Takai $Y$, Okumura $Y$, Baba M, et al. Construction of Escherichia coli K12 in-frame, single-gene knockout mutants: the Keio collection. Mol Syst Biol. 2006; 2: 2006.0008. https://doi.org/10.1038/msb4100050 PMID: 16738554

125. Lilia Perfeito, Inês Pereira M., Campos Paulo R.A, Isabel Gordo. The effect of spatial structure on adaptation in Escherichia coli. Biol Lett. 2008; 4: 57-59. https://doi.org/10.1098/rsbl.2007.0481 PMID: 18029298

126. Pinheiro J, Bates D, DebRoy S, Sarkar D, R Core Team. nlme: Linear and Nonlinear Mixed Effects Models. 2019. Available from: https://CRAN.R-project.org/package=nlme

127. R Core Team. R: A Language and Environment for Statistical Computing. Vienna, Austria: R Foundation for Statistical Computing; 2018. Available from: https://www.R-project.org/

128. Wolfram Research. Mathematica [software]. Champaign, Illinois: Wolfram Research; 2018. Available from: https://www.wolfram.com/mathematica/

129. Keseler IM, Mackie A, Santos-Zavaleta A, Billington R, Bonavides-Martínez C, Caspi R, et al. The EcoCyc database: reflecting new knowledge about Escherichia coli K-12. Nucleic Acids Res. 2017; 45: D543-D550. https://doi.org/10.1093/nar/gkw1003 PMID: 27899573

130. Price MN, Wetmore KM, Waters RJ, Callaghan M, Ray J, Liu H, et al. Mutant phenotypes for thousands of bacterial genes of unknown function. Nature. 2018; 557: 503. https://doi.org/10.1038/ s41586-018-0124-0 PMID: 29769716

131. Al Mamun AAM, Lombardo M-J, Shee C, Lisewski AM, Gonzalez C, Lin D, et al. Identity and Function of a Large Gene Network Underlying Mutagenic Repair of DNA Breaks. Science. 2012; 338: 13441348. https://doi.org/10.1126/science.1226683 PMID: 23224554

132. Horst JP, Wu TH, Marinus MG. Escherichia coli mutator genes. Trends Microbiol. 1999; 7: 29-36. https://doi.org/10.1016/s0966-842x(98)01424-3 PMID: 10068995 
133. Turrientes M-C, Baquero F, Levin BR, Martínez J-L, Ripoll A, González-Alba J-M, et al. Normal mutation rate variants arise in a Mutator (Mut S) Escherichia coli population. PLoS ONE. 2013; 8: e72963. https://doi.org/10.1371/journal.pone.0072963 PMID: 24069167

134. Huerta-Cepas J, Szklarczyk D, Forslund K, Cook H, Heller D, Walter MC, et al. eggNOG 4.5: a hierarchical orthology framework with improved functional annotations for eukaryotic, prokaryotic and viral sequences. Nucleic Acids Res. 2016; 44: D286-D293. https://doi.org/10.1093/nar/gkv1248 PMID: 26582926 